\title{
Amphetamines promote mitochondrial dysfunction and DNA damage in pulmonary hypertension
}

Pin-I Chen, Aiqin Cao, Kazuya Miyagawa, Nancy F. Tojais, Jan K. Hennigs, Caiyun G. Li, Nathaly M. Sweeney, Audrey S. Inglis, Lingli Wang, Dan Li, Matthew Ye, Brian J. Feldman, and Marlene Rabinovitch

Department of Pediatrics, Vera Moulton Wall Center for Pulmonary Vascular Diseases, and Cardiovascular Institute, Stanford University School of Medicine, Stanford, California, USA.

\begin{abstract}
Amphetamine (AMPH) or methamphetamine (METH) abuse can cause oxidative damage and is a risk factor for diseases including pulmonary arterial hypertension (PAH). Pulmonary artery endothelial cells (PAECs) from AMPH-associated-PAH patients show DNA damage as judged by $\gamma \mathrm{H} 2 \mathrm{AX}$ foci and DNA comet tails. We therefore hypothesized that AMPH induces DNA damage and vascular pathology by interfering with normal adaptation to an environmental perturbation causing oxidative stress. Consistent with this, we found that AMPH alone does not cause DNA damage in normoxic PAECs, but greatly amplifies DNA damage in hypoxic PAECs. The mechanism involves AMPH activation of protein phosphatase $2 A$, which potentiates inhibition of Akt. This increases sirtuin 1, causing deacetylation and degradation of HIF1 $\alpha$, thereby impairing its transcriptional activity, resulting in a reduction in pyruvate dehydrogenase kinase 1 and impaired cytochrome $c$ oxidase 4 isoform switch. Mitochondrial oxidative phosphorylation is inappropriately enhanced and, as a result of impaired electron transport and mitochondrial ROS increase, caspase- 3 is activated and DNA damage is induced. In mice given binge doses of METH followed by hypoxia, HIF1 $\alpha$ is suppressed and pulmonary artery DNA damage foci are associated with worse pulmonary vascular remodeling. Thus, chronic AMPH/METH can induce DNA damage associated with vascular disease by subverting the adaptive responses to oxidative stress.
\end{abstract}

Conflict of interest: The authors have declared that no conflict of interest exists.

Submitted: August 30, 2016 Accepted: December 6, 2016 Published: January 26, 2017

Reference information: JCI Insight. 2017;2(2):e90427. doi:10.1172/ji.insight.90427.

\section{Introduction}

Amphetamine (AMPH) and its chemical derivative methamphetamine (METH) are synthetic stimulants that increase catecholamine concentration in the central and peripheral nervous systems. Much of the research related to these agents has focused on their adverse effects on mental health and neurotoxicity, assessed by degeneration of dopaminergic and serotonergic terminals, and activation of astroglial and microglial cells in the brain (1). Studies using animal models have shown that oxidative damage contributes to AMPH-induced neurotoxicity (2-4), and that free radical scavengers and antioxidants, or overexpression of antioxidant enzymes, can attenuate the neurotoxic effects of AMPH $(5,6)$. However, little is known mechanistically about how AMPH impairs adaptation to cellular stresses that are potentially genotoxic, such as oxidant injury, and that can cause cellular dysfunction leading to disease.

$\mathrm{AMPH}$ and anorexigens are risk factors for pulmonary arterial hypertension (PAH) (7-9), a severe disease in which a progressive increase in resistance to blood flow through the lung can culminate in right heart failure and death in the absence of lung transplantation. The pathological features of PAH include accumulation of DNA damage and genomic instability in pulmonary artery endothelial cells (PAECs) $(10,11)$, loss of the precapillary arteries due to PAEC apoptosis, abnormal release of PAEC mediators that stimulate the recruitment of inflammatory cells, and the exuberant proliferation of smooth muscle-like cells, that result in occlusion and stiffening of the pulmonary arteries (reviewed in ref. 12). The mechanism linking AMPH and anorexigens to PAH was attributed to their pharmacologic similarity to serotonin, a monoamine that is both a potent vasoconstrictor and mitogen for smooth muscle cells $(9,13)$. Although AMPH/METH abusers have a 3-fold increased risk of developing PAH (reviewed in ref. 14), mechanisms causing individual susceptibility remain unclear and combinatorial interactions with environmental stimuli and/or genetic factors, while likely, have not been investigated. 
Table 1. Characteristics of drug and toxin-associated PAH (D\&T-PAH) patients that were the source of PAECs used in the experiments in Figure 1

\begin{tabular}{|c|c|c|c|c|c|c|c|c|c|c|}
\hline ID & $\begin{array}{l}\text { PAEC } \\
\text { Type }\end{array}$ & $\begin{array}{l}\text { Age } \\
\text { (yr) }\end{array}$ & Gender & Race/Ethnicity & Diagnosis & $\operatorname{PAP}(s / d / m)^{A}$ & $\begin{array}{l}\text { PVR } \\
\text { (WU) }\end{array}$ & $\begin{array}{c}\text { 6MW } \\
(\mathrm{m})\end{array}$ & PAH Medications & $\begin{array}{l}\text { Known BMPR2 or } \\
\text { SMAD Mutation }\end{array}$ \\
\hline PAH-1 & MVEC & 53 & $\mathrm{~F}$ & $\begin{array}{l}\text { White/Non- } \\
\text { Hispanic }\end{array}$ & $\begin{array}{c}\text { APAH } \\
\text { Drugs and Toxins } \\
\text { (Amphetamine) }\end{array}$ & $100 / 45 / 68$ & 15.99 & 335 & $\begin{array}{l}\text { sildenafil bosentan } \\
\text { treprostinil iloprost }\end{array}$ & No \\
\hline РАH-2 & LPAEC & 41 & $\mathrm{~F}$ & $\begin{array}{l}\text { White/Non- } \\
\text { Hispanic }\end{array}$ & $\begin{array}{c}\text { APAH } \\
\text { Drugs and toxins } \\
\text { (Anorexigens) }\end{array}$ & $86 / 35 / 55$ & 9.44 & 271 & $\begin{array}{c}\text { epoprostenol } \\
\text { bosentan, iloprost } \\
\text { sildenafil }\end{array}$ & No \\
\hline PAH-4 & LPAEC & 42 & $\mathrm{~F}$ & $\begin{array}{l}\text { Asian/Non- } \\
\text { Hispanic }\end{array}$ & $\begin{array}{l}\text { APAH Drugs and Toxins } \\
\text { (Methamphetamine) }\end{array}$ & $75 / 31 / 51$ & 9.80 & 520 & $\begin{array}{c}\text { riociguat ambrisentan } \\
\text { treprostinil }\end{array}$ & Unknown \\
\hline PAH-5 & SPAEC & 42 & $F$ & $\begin{array}{l}\text { White/Non- } \\
\text { Hispanic }\end{array}$ & $\begin{array}{l}\text { APAH Drugs and Toxins } \\
\text { (Methamphetamine, } \\
\text { cocaine, anorexigens) }\end{array}$ & $74 / 37 / 52$ & $N / A$ & N/A & $\begin{array}{l}\text { tadalafil ambrisentan } \\
\text { treprostinil }\end{array}$ & No \\
\hline
\end{tabular}

${ }^{A}$ Hemodynamic data of pulmonary arterial hypertension (PAH) patients were obtained from catheterization studies performed closest to transplantation. PAH medications are listed according to total drug exposure during treatment period of follow-up, not necessarily in combination. BMPR2, bone morphogenetic protein receptor type II; PAEC, pulmonary artery endothelial cell; LPAEC, large PAEC; SPAEC, small PAEC; MVEC, microvessel endothelial cell; PAP, pulmonary artery pressure $(\mathrm{mmHg})$ (s, systolic; d, diastolic; $\mathrm{m}$, mean); WU, Wood Units (Baseline Fick PVR); 6 MW, distance (m) walked in 6 minutes. N/A, not available.

Low oxygen tension (hypoxia) is an environmental stress that occurs under both physiological and pathological conditions, including high altitude, sleep apnea, anemia, ischemia, and obstructive/restrictive lung diseases. Alveolar hypoxia is a potent stimulus for pulmonary vasoconstriction (15) that can lead to the development of chronic pulmonary hypertension, and is known to synergize with serotonin in worsening pulmonary vascular disease (16). Chronic or severe hypoxia can result in an imbalance between antioxidant capacity and oxidants. Hypoxia can also suppress DNA repair, resulting in genomic instability and increased rates of mutagenesis, particularly in actively proliferating cells (17), perhaps accounting for somatic mutations described in PAH PAECs $(10,11)$. Understanding how aberrant molecular signaling causes DNA damage as an early event that initiates vascular remodeling is the subject of ongoing investigation (18). For example, the inflammatory mediator TNF- $\alpha$ (19), and genetic deficiency of BMPR2 or TopBP1 $(20,21)$ can both promote DNA damage and impair endothelial cell proliferation and survival. Recently, we demonstrated that oxidative stress caused by reoxygenation after hypoxia combined with BMPR2 deficiency induced a mitochondrial DNA deletion, elevated caspase-3/7 activity in PAECs, and impaired reversal of pulmonary hypertension (22).

These studies led us to hypothesize that, in the context of an environmental stress such as hypoxia, AMPH exposure promotes accumulation of DNA damage and more severe remodeling of the pulmonary vasculature. We now describe a process whereby AMPH subverts the HIF1 $\alpha$-mediated metabolic response to hypoxic exposure, causing abnormal mitochondrial ROS production, activation of caspase-3, and DNA damage in PAECs. Reproducing this adverse response for several weeks in a mouse induced DNA damage in vascular cells and caused greater adverse remodeling of the pulmonary arteries.

\section{Results}

PAECS from AMPH- and anorexigen-PAH patients show persistent DNA damage. To establish the relationship between AMPH abuse and DNA damage in target tissues, we harvested PAECs from lungs removed at transplant from 3 AMPH-PAH and 1 anorexigen-PAH patient (under the umbrella clinical classification, drug- and toxin-associated PAH; D\&T-PAH), and from 4 unused donor control lungs (Tables 1 and 2 give demographic and clinical details). We found greater evidence of DNA damage in the PAH patients' PAECs, as judged by increased comet tails in a gel electrophoresis assay (Figure 1A) and $\gamma \mathrm{H} 2 \mathrm{AX}$ foci (Figure 1B) at baseline that further accumulated upon exposure to doxorubicin (Dox), a genotoxin that causes DNA double-stranded breaks (23). Dox withdrawal allowed these cells to recover, yet greater DNA damage in D\&T-PAH patient PAECs persisted. 
A

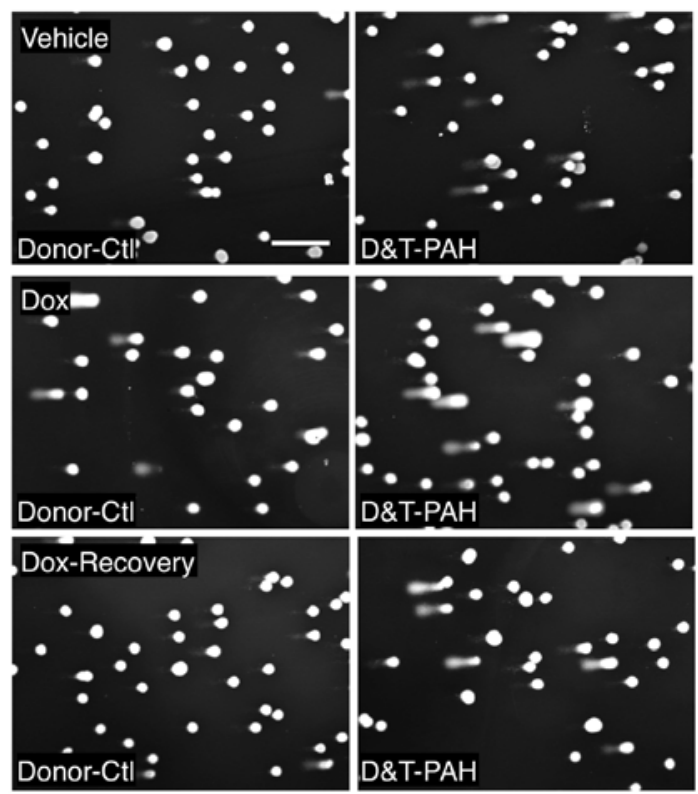

口Donor Control 口D\&T-PAH

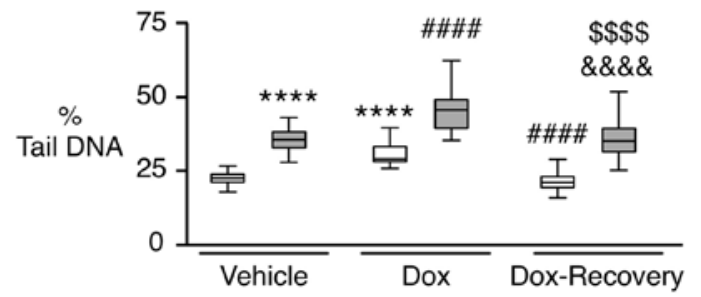

B
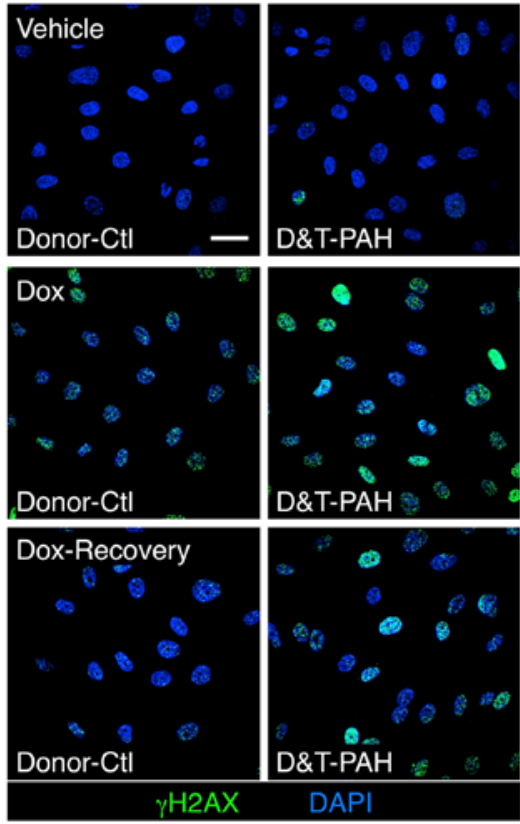

$\square$ Donor Control 口D\&T-PAH

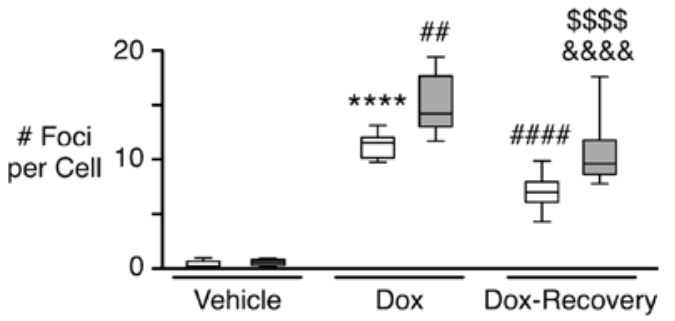

Figure 1. Unrepaired DNA damage in PAECs isolated from D\&T-PAH patients. Pulmonary artery endothelial cells (PAECs) were isolated from lung explants of unused control donor (Donor-Ctl, $n=4$ ) and from patients with pulmonary arterial hypertension (PAH) associated with amphetamine (AMPH), methamphetamine (METH) or anorexigens (D\&T-PAH, $n=4$, including 3 AMPH-PAH and 1 anorexigen-PAH), as described under Methods. PAECs were treated in full medium with vehicle (Veh) or doxorubicin (Dox) for 6 hours. A separate set of Dox-treated cells was allowed to recover (Dox-recovery) for 12 hours in A, or for 6 hours in B. (A) Cells were analyzed by alkaline comet assay. Representative images show intact DNA (comet head), and comet tails, indicative of damaged DNA. The percentage of DNA in the tail of 100 to 150 cells per subject was analyzed using Image). Scale bar: $60 \mu \mathrm{m}$. (B) Cells were immunostained for $\gamma \mathrm{H} 2 \mathrm{AX}$ and DAPI to assess DNA damage. Representative images show DNA damage foci in the nuclei. Scale bar: $30 \mu \mathrm{m}$. Number of $\gamma \mathrm{H} 2 \mathrm{AX}$ foci per cell was quantified using Image). Ten to 15 confocal images were assessed per subject. Boxand-whisker plots represent values within the interquartile range (boxes) and the minimum to maximum (whiskers). The line within the box shows the median. $n=4$, Donor-CtI PAECs, and $n=4$, D\&T-PAH PAECs, each line of PAEC has 3-4 replicate images. ${ }^{* * * *} P<0.0001$ vs. Donor-Ctl+Veh; ${ }^{\# \# P} P 0.005$ and ${ }^{\# \# \# P} P 0.0001$ vs. Donor-Ctl+Dox; \&\&\&\& $P<0.0001$ vs. D\&T-PAH+Dox; ${ }^{\$ \$ \$ \$} P<0.0001$ vs. Donor-Ctl+Dox-recovery, by 2-way ANOVA, Bonferroni's post-test.

AMPH exaggerates DNA damage induced by Dox and by hypoxia. To determine whether AMPH could promote or exaggerate DNA damage, we treated commercially available PAECs with AMPH alone or together with Dox or hypoxia $\left(0.5 \% \mathrm{O}_{2}\right)$, that can initiate DNA damage via replication arrest (17). While AMPH alone did not induce DNA damage in cells cultured in full serum (Figure 2D), it exaggerated DNA damage induced by Dox in a dose-dependent manner, as judged by $\gamma \mathrm{H} 2 \mathrm{AX}$ immunoblots (Figure $2 \mathrm{~A}$ ). We also showed an increase in comet tails (Figure 2B) and in $\gamma \mathrm{H} 2 \mathrm{AX}$ foci formation (Figure 2C) when PAECs were treated daily with $1 \mathrm{mM}$ AMPH for 3 days followed by Dox for just 6 hours. Similarly, AMPH exaggerated DNA damage induced by 48-hour hypoxia in a dose-dependent manner, as judged by $\gamma \mathrm{H} 2 \mathrm{AX}$ immunoblot (Figure 2D) and by comet tails (Figure 2E). We next related the mechanism of AMPH-induced DNA damage in hypoxia to activation of caspase-3 by low phospho-Akt (p-Akt). 

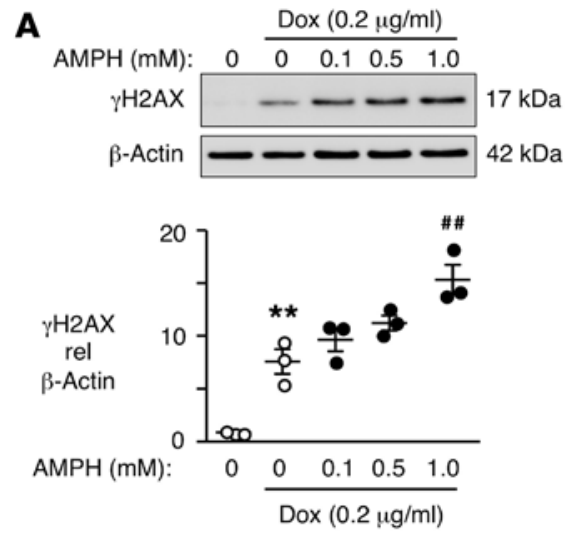

D

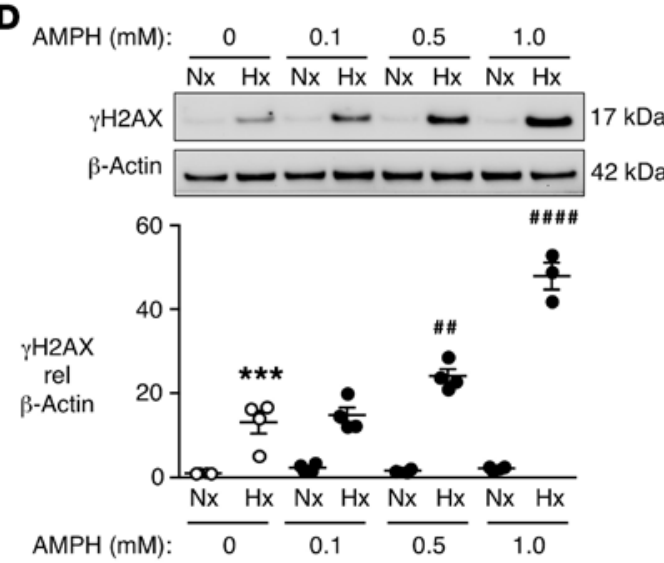

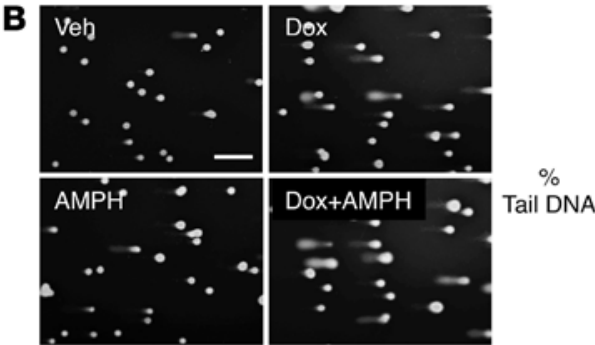
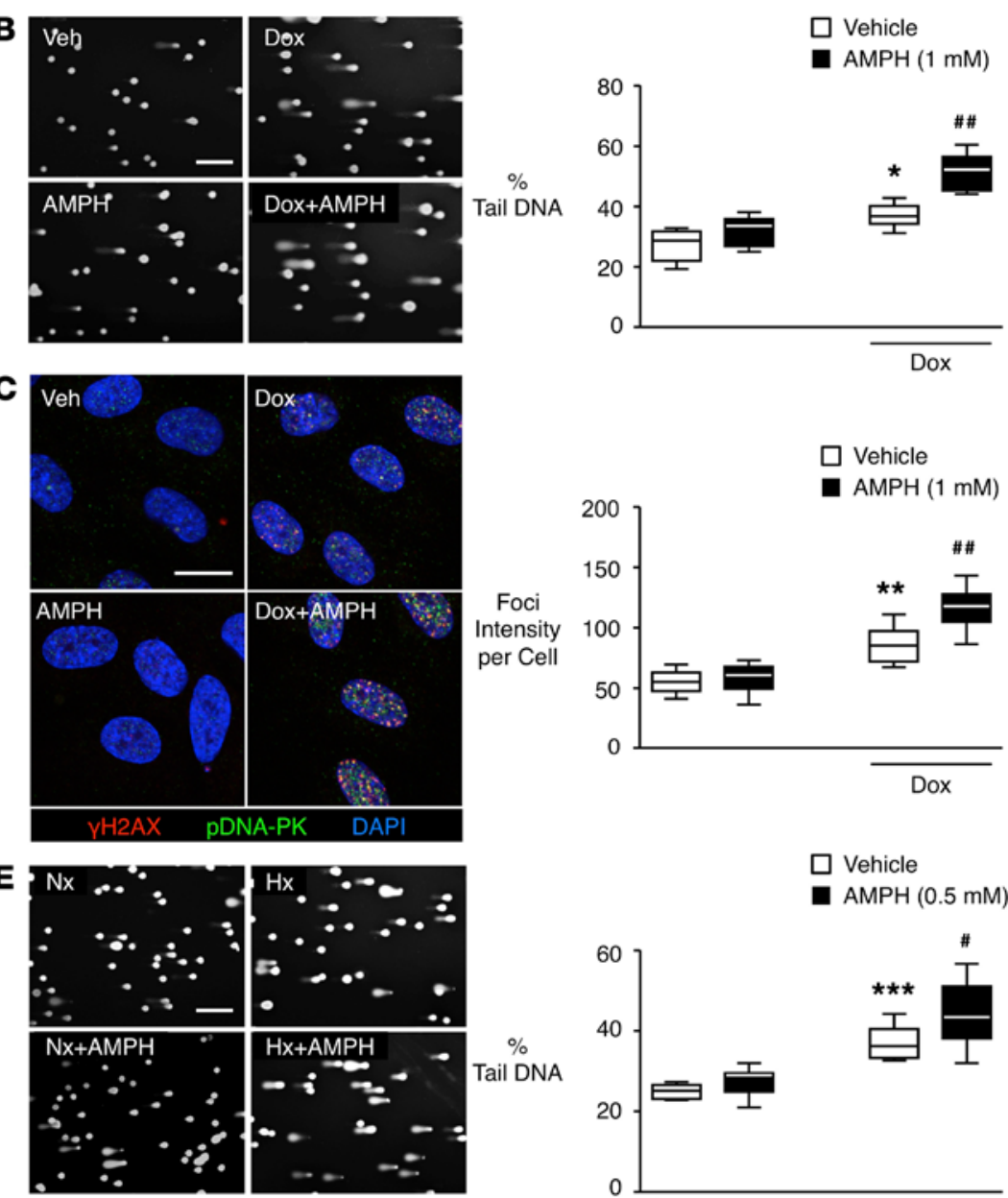

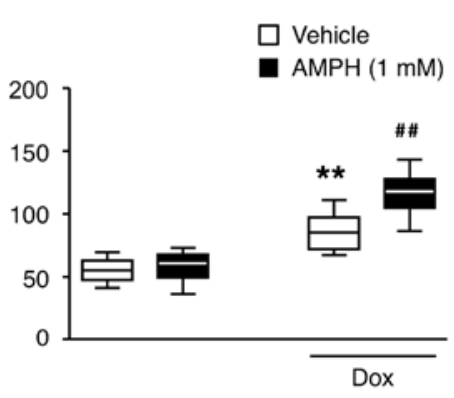

$\square$ Vehicle

AMPH $(0.5 \mathrm{mM})$

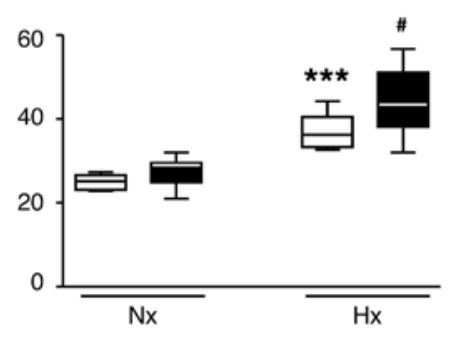

Figure 2. Amphetamine exacerbates genotoxic stress-induced DNA damage. (A) Commercially available human pulmonary artery endothelial cells (PAECs) were treated in full medium with indicated doses of amphetamine (AMPH) daily for 3 days, and then stimulated with doxorubicin (Dox, 0.2 $\mu \mathrm{g} / \mathrm{ml}$ ) for 24 hours in the presence of vehicle (Veh) or AMPH. Cell lysates were immunoblotted for $\gamma \mathrm{H} 2 \mathrm{AX}$ and $\beta$-actin (for normalization). (B) PAECs were treated with $1 \mathrm{mM} \mathrm{AMPH}$ daily for 3 days, and then stimulated with Dox in the presence of Veh or AMPH for 6 hours. Cells were analyzed by comet assay as described for Figure 1. Scale bar: $60 \mu \mathrm{m}$. (C) Cells treated as in B were immunostained for $\gamma \mathrm{H} 2 \mathrm{AX}$ (red), pDNA-PK (green), and DAPI (blue). Ten to 15 confocal images with at least 40 cells per image per condition were analyzed using Image). Scale bar: $15 \mu \mathrm{m}$. (D) PAECs were treated daily for 3 days with the indicated dose of $\mathrm{AMPH}$, and then cultured with Veh or AMPH under $0.5 \% \mathrm{O}$, hypoxia (Hx) or normoxia (Nx) for 48 hours. Cell lysates were assayed as in A. (E) Comet assay of PAECs treated with $0.5 \mathrm{mM} \mathrm{AMPH}$ and Hx/Nx as in $\mathbf{D}$. Scale bar: $60 \mu \mathrm{m}$. (A and D) Dot plots represent mean \pm SEM, $n=3-4$. (B, C, and E) Box-and-whisker plots represent values within the interquartile range (boxes) and the minimum to maximum (whiskers). The line within the box shows the median. $n=3$ independent experiments with 3 to 5 replicates per experiment. $(\mathbf{A}-\mathbf{C}){ }^{*} P<0.05,{ }^{* *} P<0.005$

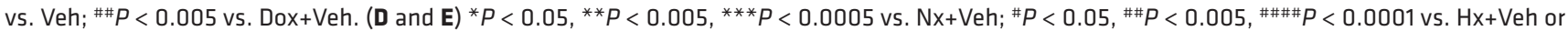
$\mathrm{Vec}+\mathrm{Hx}+\mathrm{Veh} ;{ }^{\&} P<0.05, \& \& P<0.005$, \&\&\&\& $P<0.0001 \mathrm{vs}$. $\mathrm{Hx}+\mathrm{AMPH}$ or $\mathrm{Vec}+\mathrm{Hx}+\mathrm{AMPH}$, by 2 -way ANOVA, Bonferroni's post-test.

In separate experiments using 24-hour serum withdrawal as a stimulus, we determined that AMPH (Supplemental Figure 1A; supplemental material available online with this article; doi:10.1172/jci.insight.90427DS1) and METH (Supplemental Figure 1B) promote caspase-3/ 7 activation in a dose-dependent manner using concentrations relevant to the clinical setting $(24,25)$. We then identified an AMPH-mediated reduction in Akt phosphorylation (Supplemental Figure 1C) that we attributed to high protein phosphatase (PP1 and PP2) activities, since it was reversed by PP1 and PP2 inhibition with okadaic acid but not by restoring PI(3,4,5)P3 levels with the phosphatase and tensin homolog (PTEN) inhibitor (VO-OHpic; Supplemental Figure 1D). Consistent with these results, we detected enhanced PP2A activity in PAECs treated with AMPH (Supplemental Figure $1 \mathrm{E})$. This feature is in keeping with previous studies showing that AMPH-induced activation of dopamine receptors in the mouse striatum results in a complex between Akt, $\beta$-arrestin2, and PP2A (26).

We then showed in PAECs in full serum that the AMPH-mediated reduction in p-Akt was further decreased under conditions of hypoxia (Figure 3A) or Dox (Supplemental Figure 2A) and this greatly 
A
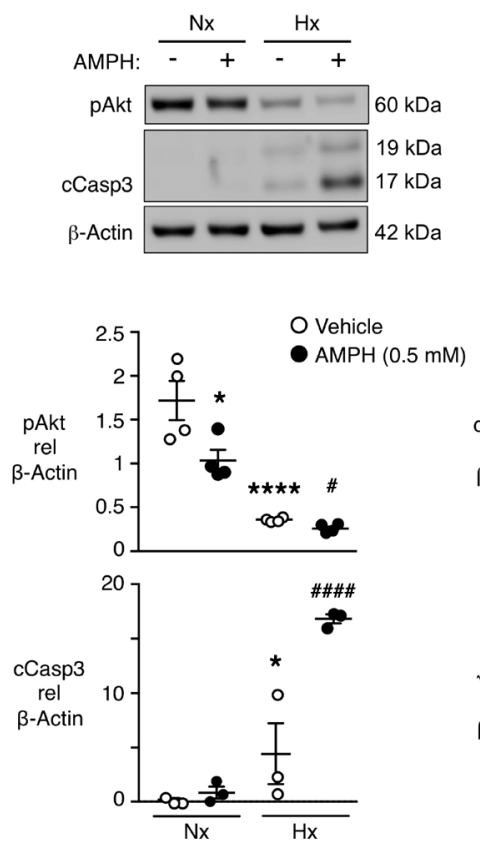

B
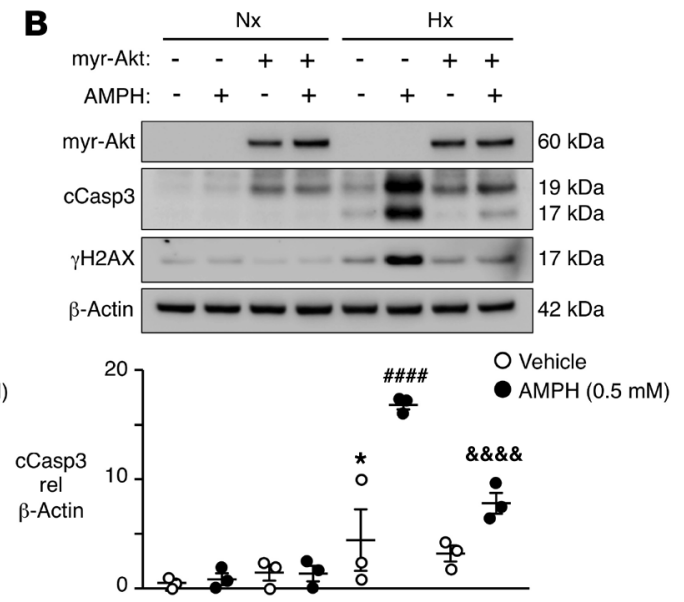

C

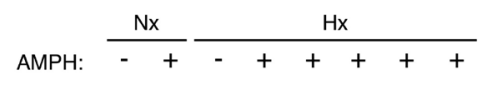

$\operatorname{zVAD}(\mu \mathrm{M}): \quad-\quad-\quad-\quad 0 \quad 5 \quad 10 \quad 20 \quad 40$

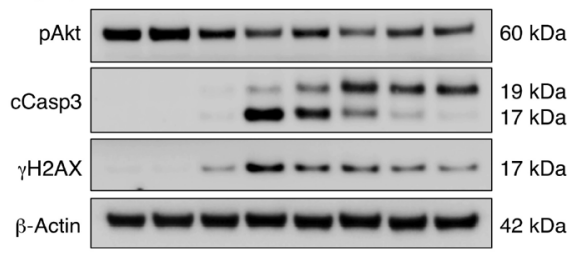

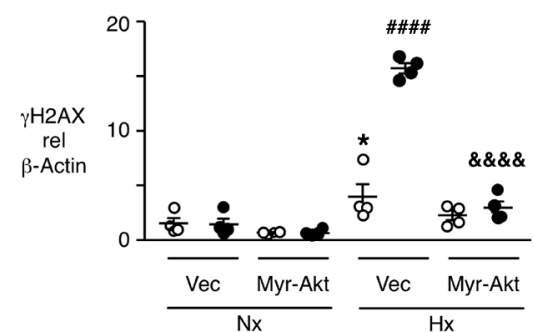

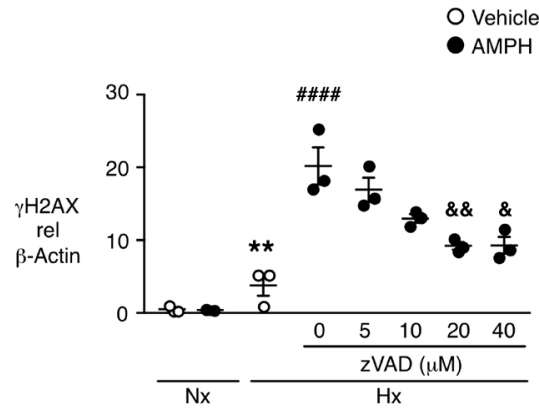

Figure 3. Amphetamine exaggerates hypoxia-induced DNA damage in a p-Akt- and caspase-3-dependent manner. (A) Pulmonary artery endothelial cells (PAECs) were treated daily for 3 days with the indicated $0.5 \mathrm{mM}$ amphetamine (AMPH), and then cultured with vehicle (Veh) or AMPH under $0.5 \% \mathrm{O}_{2}$ hypoxia ( $\mathrm{Hx}$ ) or normoxia ( $\mathrm{Nx}$ ) for 48 hours, immunoblotted for p-Akt, cleaved caspase-3 (cCasp3), and $\beta$-actin (loading control). (B) PAECs were transfected with vector (Vec) or HA-tagged myristoylated Akt1 (myr-Akt) construct. Twenty-four hours after transfection, cells were treated with AMPH and Hx/Nx as in $\mathbf{A}$. Cell lysates were immunoblotted for $\gamma \mathrm{H} 2 \mathrm{AX}$, cCasp3, $\beta$-actin (for normalization), and HA, to verify the expression of HA-myr-Akt. (C) PAECs were treated as in $\mathbf{A}$ together with the indicated concentrations of zVAD-FMK, and lysates were immunoblotted for $\gamma \mathrm{H} 2 \mathrm{AX}, \mathrm{cC}$ asp3, $\beta$-actin (loading control), and $p$-Akt. Dot plots represent mean \pm SEM, $n=3-4$. (A-C) ${ }^{*} P<0.05,{ }^{* *} P<0.005$, ${ }^{* * * *} P<0.0001$ vs. Nx+Veh; ${ }^{*} P<0.05$, \#\# $P<0.005$, \#\#\# $P<0.0001$ vs. $\mathrm{Hx}+\mathrm{Veh}$ or Vec $+\mathrm{Hx}+\mathrm{Veh} ;{ }^{\&} \mathrm{P}<0.05$, \&\& $P<0.005$, \&\&\&\& $P<0.0001$ vs. Hx+AMPH or Vec+Hx+AMPH; by 2-way ANOVA, Bonferroni's post-test.

increased both DNA damage assessed by $\gamma \mathrm{H} 2 \mathrm{AX}$ and active caspase-3 (Figure 3B and Supplemental Figure 2A). Moreover, PAECs transfected with constitutively active myristoylated Akt (myr-Akt) (Figure 3B), or treated with a pan-caspase inhibitor (zVAD-FMK) (Figure 3C), reversed the AMPH-induced increase in $\gamma \mathrm{H} 2 \mathrm{AX}$ and active caspase-3. A similar response to myr-Akt was seen with Dox (Supplemental Figure 2B).

Amplified hypoxia-induced DNA damage by AMPH in PAECs is related to sirtuin 1. Since DNA accessibility can influence propensity to damage and repair (27), we determined whether AMPH augments the hypoxiaassociated histone deacetylation previously described (28). Indeed, AMPH enhanced deacetylation of both histone 3 and 4 under hypoxia (Figure 4A). We therefore screened histone deacetylases (HDACs) by mRNA levels, and found that AMPH upregulated many HDACs in hypoxia, including members of the sirtuin (SIRT) family, particularly SIRT1 (Figure 4B). Using either the SIRT1/2 inhibitor, sirtinol (Figure 4C), or the panHDAC inhibitor, trichostatin A (TSA) (Supplemental Figure 3A), we abrogated the AMPH-exaggerated $\gamma \mathrm{H} 2 \mathrm{AX}$ and caspase-3 activation. Similar results were evident in cells treated with SIRT1 siRNA (Figure 4D).

As reduced p-Akt was not, however, rescued by SIRT1 siRNA (Figure 4D), we reasoned that low p-Akt could be regulating elevated SIRT1. Phosphorylation of SIRT1 at $\operatorname{Ser}^{46}$ (in mouse) or $\mathrm{Ser}^{47}$ (in human) results in its ubiquitination and proteasome-dependent degradation (29). As JUN N-terminal kinase (JNK) mediates the phosphorylation of SIRT1 (30), we reasoned that this might also occur in response to p-Akt. Indeed, we found that at 24 hours, SIRT1 was increased in hypoxia by AMPH in association with very low levels of p-Akt and p-SIRT1, but levels of p-JNK were unchanged (Figure 5A). Moreover, expression of myr-Akt reversed the AMPH-induced increase in SIRT1 during hypoxia, providing further evidence that SIRT1 is destabilized by p-Akt (Figure 5B). It is interesting that SIRT1 mRNA levels also were no longer significantly elevated (Supplemental Figure 3B), suggesting that Akt might be coordinating SIRT1 mRNA $(31,32)$ and protein levels, as has been shown with other signaling molecules such as MAPK-activated protein kinase 2 (33). 
A
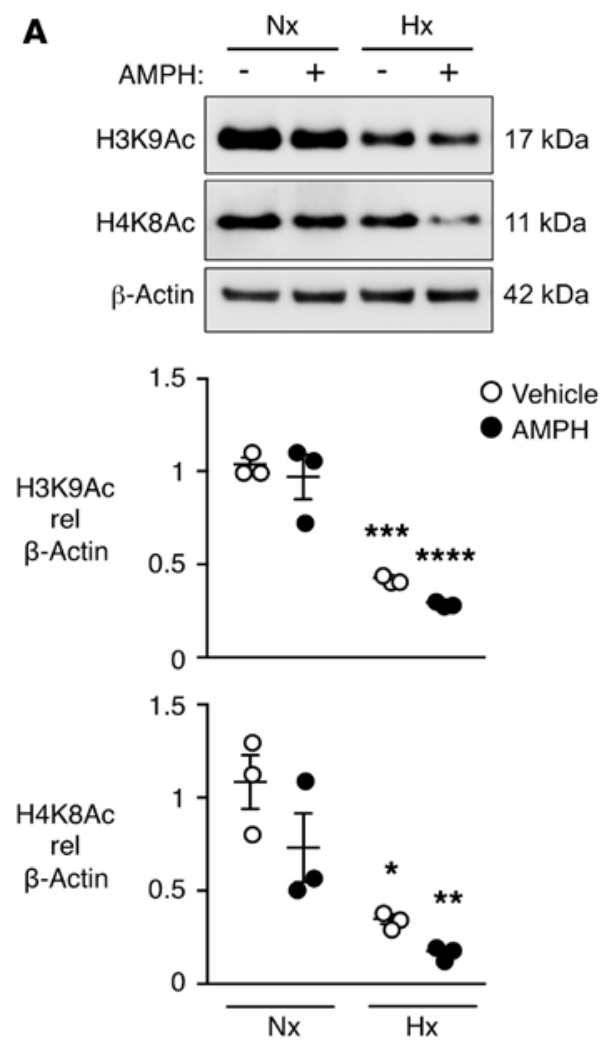

c
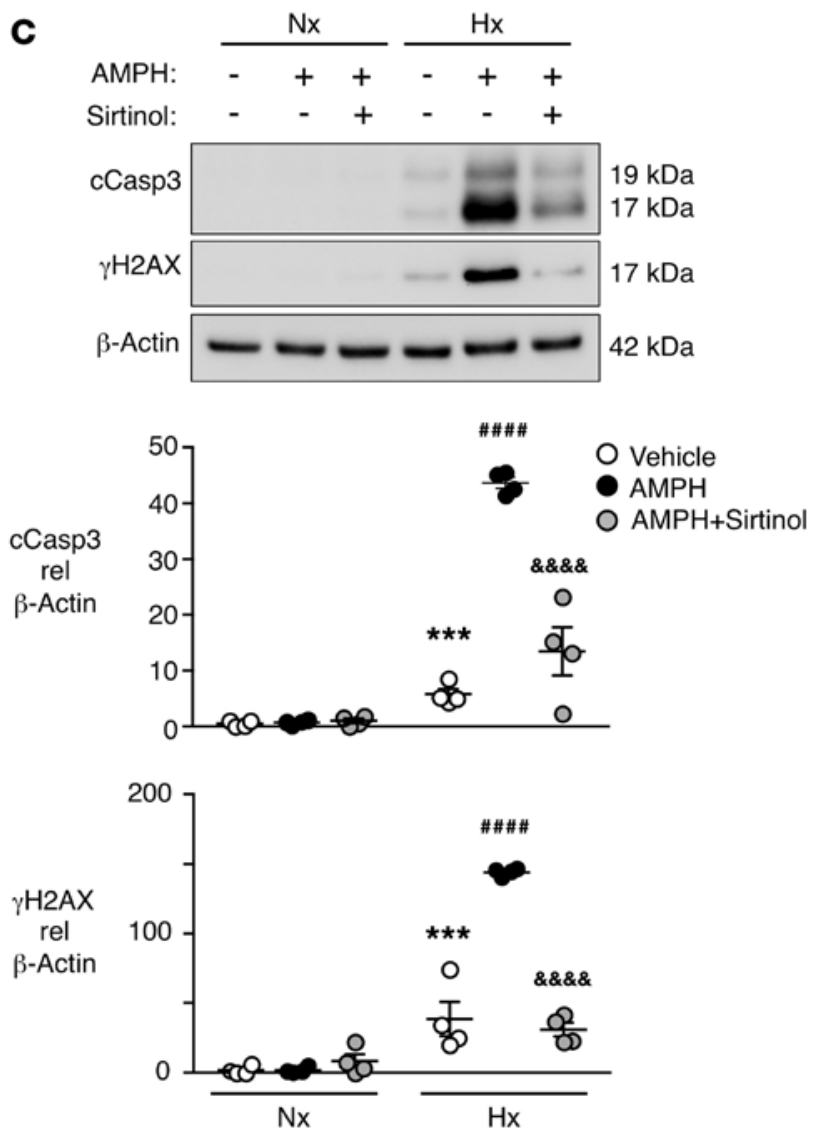

B

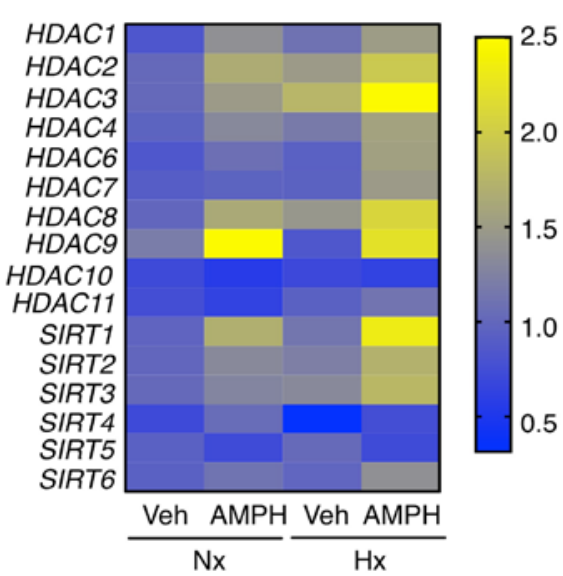

D
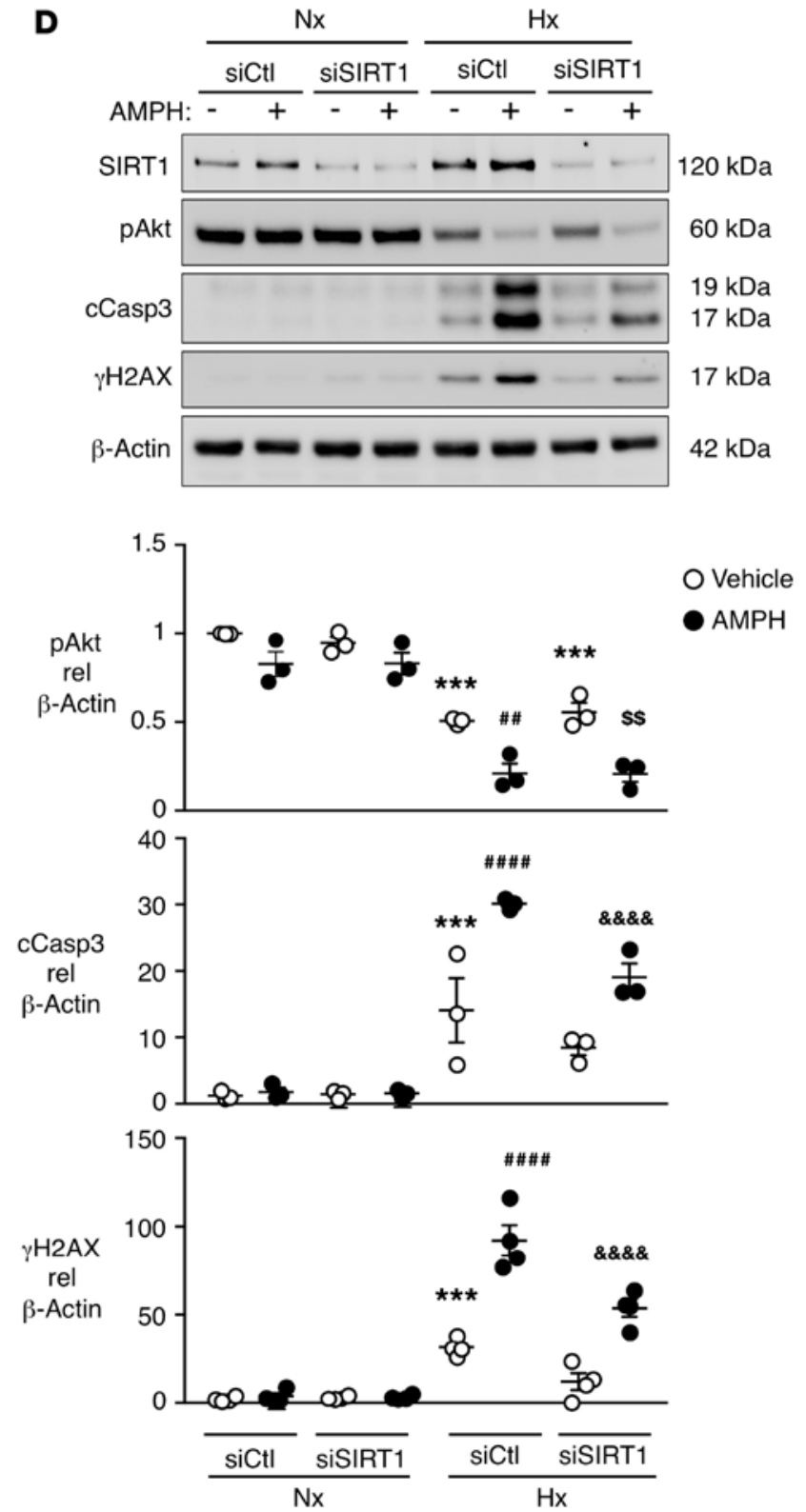
Figure 4. Amphetamine-induced SIRT1 stabilization under hypoxia exaggerates caspase-3 activation and DNA damage (see also Supplemental Figure 3). Pulmonary artery endothelial cells (PAECs) were treated daily with $0.5 \mathrm{mM}$ amphetamine (AMPH) for 3 days and then cultured with vehicle (Veh) or AMPH under normoxia (Nx) or hypoxia (Hx) for (A) 48 hours or (B) 8 hours. Cells were (A) immunoblotted for acetylated histone 3 (H3K9Ac), histone 4 (H4K8Ac), and $\beta$-actin (for normalization) or (B) extracted for RNA and then screened for histone deacetylase (HDAC) expression via quantitative real-time PCR (qRT-PCR). qRT-PCR results are displayed as a heatmap with blue to yellow indicating low to high levels relative to Veh for each condition. (C) PAECs were treated as described in $\mathbf{A}$, with or without sirtinol $(10 \mu \mathrm{M})$ during the treatment. Cell lysates were immunoblotted for $\gamma \mathrm{H} 2 \mathrm{AX}$, cleaved caspase-3 (cCasp3), and $\beta$-actin (loading control). (D) PAECs were transfected with $20 \mathrm{nM}$ control (siCtI) or SIRT1 (siSIRT1) siRNA. Twenty-four hours after transfection, cells were treated as in $\mathbf{A}$ and analyzed for SIRT1, p-Akt, $\gamma \mathrm{H} 2 \mathrm{AX}$, cCasp3, and $\beta$-actin (loading control). Dot plots represent mean $\pm \mathrm{SEM}, n=3-4$. ${ }^{*} P<0.05$, ${ }^{* *} P<0.005$, ${ }^{* *} P<0.0005$, ${ }^{* * * *} P<0.0001$ vs. (siCtl or Vec) $+\mathrm{Nx}+\mathrm{Veh}$; ${ }^{\# \#} P<0.005$, ${ }^{* \# \#} P<0.0001$ vs. (siCtl or Vec) $+\mathrm{Hx}+\mathrm{Veh}$; ${ }^{\$ \$} P<0.005 \mathrm{vs.}$ siSIRT1+Hx+Veh; ${ }^{\&} P<0.05$, \&\&\&\& $P<0.0005$ vs. (siCtl or Vec) $+\mathrm{Hx}+\mathrm{AMPH}$; by 2 -way ANOVA, Bonferroni's post-test.

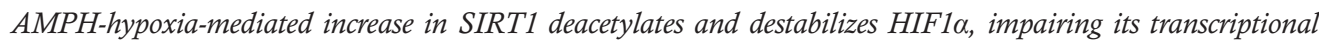
activity. While inhibition of SIRT1 by siRNA reversed AMPH-exaggerated $\gamma \mathrm{H} 2 \mathrm{AX}$ and caspase-3 activation, it did not reverse the deacetylation of histones (Supplemental Figure 3C) nor did expression of myr-Akt (Supplemental Figure 3D). This suggests that histone deacetylation in hypoxia is not mediated by the elevation in SIRT1, and does not account for the AMPH-mediated amplification of DNA damage and caspase- 3 activation.

In addition to histones, SIRT1 deacetylates various transcription factors critical for cell survival and metabolism, such as p53, FOXOs, and HIFs (34). Under hypoxia, NAD ${ }^{+}$deficiency decreases SIRT1 activity, enhancing acetylation that stabilizes HIF1 $\alpha$ (35). We found that HIF1 $\alpha$ levels were reduced by AMPH under hypoxia (Figure 6A). To verify that AMPH leads to deacetylation and destabilization of HIF $1 \alpha$ by SIRT1, we treated PAECs with AMPH alone or together with sirtinol, and cell lysates were immunoprecipitated with anti-acetyl-lysine antibodies and probed for HIF1 $\alpha$. The data show that HIF1 $\alpha$ was deacetylated when exposed to AMPH under hypoxia, and addition of sirtinol reversed the deacetylation (Figure 6B). In keeping with these results, suppressing SIRT1 with siRNA also reversed the decrease in HIF1 $\alpha$ mediated by AMPH during hypoxia (Figure 6C). To confirm that reduced HIF1 $\alpha$ is associated with impaired transcription, we carried out a HIF-response element luciferase assay and found that HIF transcriptional activity under hypoxia was reduced by AMPH, and restored by SIRT1 siRNA (Figure 6D).

We then investigated how the AMPH-mediated reduction in HIF1 $\alpha$ during hypoxia might be related to heightened caspase- 3 activity and DNA damage. HIF1 $\alpha$ transactivates genes that regulate glucose metabolism and redox homeostasis in response to reduced oxygen availability, and HIF $1 \alpha$-null cells die under prolonged hypoxia due to ROS toxicity (36). Two critical HIF1 $\alpha$-mediated adaptations prevent excessive ROS production in hypoxic cells. First, HIF1 $\alpha$ induces expression of pyruvate dehydrogenase kinase 1 (PDK1) to facilitate the transition from oxidative phosphorylation to glycolysis. Second, HIF $1 \alpha$ mediates a switch in the subunit composition of cytochrome $c$ oxidase (COX) from COX4 isoform 1 (COX4I1) to isoform 2 (COX4I2) to optimize electron transport during hypoxia (37). Consistent with reduced HIF1 $\alpha$, we found a significant increase in COX4I1 mRNA and a decrease in PDK1 mRNA by AMPH during hypoxia (Figure $6, \mathrm{E}$ and $\mathrm{F}$ ). Interestingly, rescuing HIF1 $\alpha$ activity by SIRT1 suppression reversed the elevated COX4I1 mRNA levels (Figure 6F) and the reduction in PDK1 was no longer significant (Figure 6E). Similar results were seen with COX4I1 and PDK1 protein levels (Figure 6G).

AMPH impairs the switch from oxidative phosphorylation to glycolysis under hypoxia. We next determined whether the SIRT1-mediated reduction in HIF1 $\alpha$ impairs the switch from oxidative phosphorylation to glycolysis. While hypoxic exposure of PAECs enhanced anaerobic glycolysis and reduced the oxygen consumption rate (OCR), addition of AMPH increased the glycolytic reserve by decreasing glycolysis and increased oxidative phosphorylation (basal and maximal OCR; Figure 7, A and B). Suppression of SIRT1 by sirtinol partially reversed the elevation of basal and maximal respiration rate (OCR) observed in AMPH and hypoxia (Figure 7B). However, reduced glycolysis (Figure 7B) was sustained, which may reflect an inability to fully recover a HIF1 $\alpha$-independent mechanism of glycolysis.

COX4 isoform switch is necessary to prevent excess production of ROS during hypoxia. Consistent with this, AMPH increased production of mitochondrial ROS during hypoxia (Figure 7, C and D). This excess ROS can be expected to cause opening of the mitochondrial permeability transition pore (mPTP) and leakage of ions, which decreases the mitochondrial membrane potential (Figure 7E). The increase in ROS and mitochondrial depolarization were reversed by suppressing SIRT1 activity using sirtinol (Figure 7, C-E), suggesting that restoring the COX4 isoform switch could prevent mPTP opening and the cytochrome $c$ leakage responsible for the caspase- 3 activation and DNA damage. We therefore treated cells with antimycin 
A

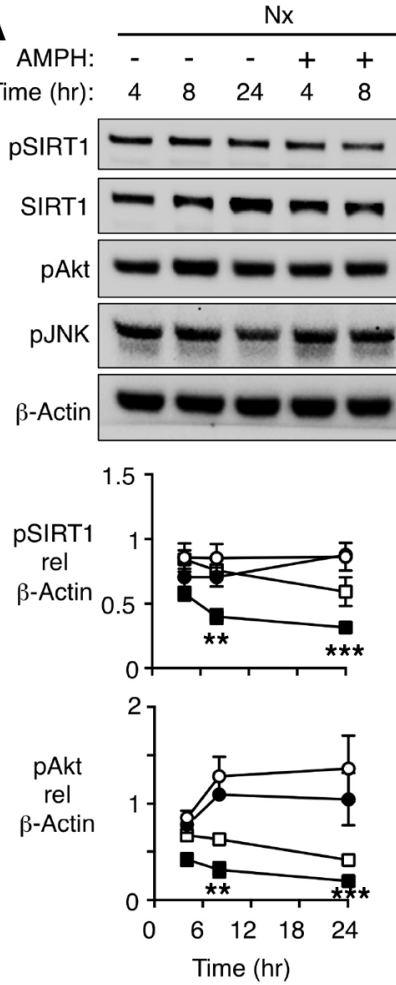

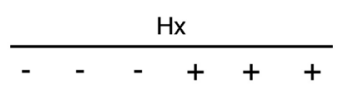

$\begin{array}{lllllll}4 & 8 & 24 & 4 & 8 & 24\end{array}$

$--\cdots-\cdots$

$\infty-\infty+\infty$

$-m-m-m$
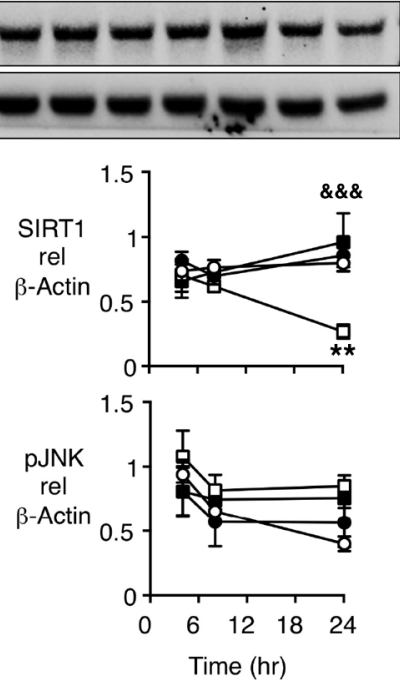

$120 \mathrm{kDa}$

$120 \mathrm{kDa}$

$60 \mathrm{kDa}$

$54 \mathrm{kDa}$

$42 \mathrm{kDa}$

$\mathrm{Nx}+$ Vehicle

$\mathrm{Nx}+\mathrm{AMPH}$

$\square \mathrm{Hx}+$ Vehicle

$\mathrm{Hx}+\mathrm{AMPH}$
B

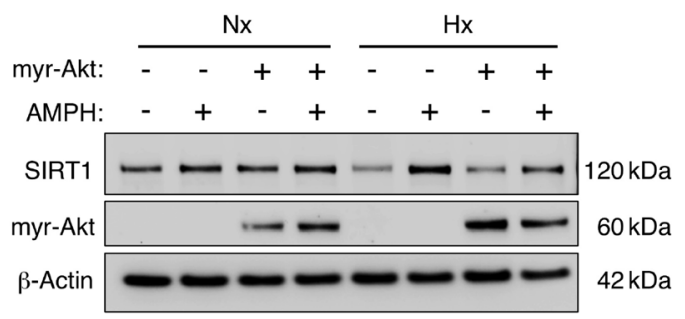

Figure 5. Suppression of p-Akt by amphetamine stabilizes SIRT1 under hypoxia (See also Supplemental Figure 3). (A) Pulmonary artery endothelial cells (PAECs) were treated with $0.5 \mathrm{mM}$ amphetamine (AMPH) for the indicated times under normoxia ( $\mathrm{Nx}$ ) or hypoxia ( $\mathrm{Hx}$ ), and cell lysates were immunoblotted for phospho-SIRT1 (p-SIRT1), SIRT1, p-Akt, p-JNK, and $\beta$-actin (loading control). (B) PAECs were transfected with vector (Vec) or HA-tagged myristoylated Akt1 (myr-Akt) construct. Twenty-four hours after transfection, cells were treated daily with $0.5 \mathrm{mM} \mathrm{AMPH}$ for 3 days and then cultured with vehicle (Veh) or AMPH under Nx or Hx for 48 hours, and SIRT1, myr-Akt, and $\beta$-actin (loading control) protein levels were analyzed by immunoblotting. Dot and line

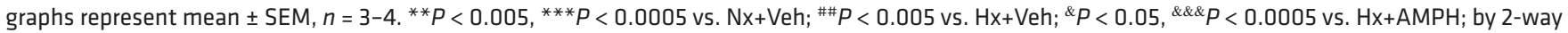
ANOVA, Bonferroni's post-test.

A (AMA), an inhibitor of mitochondrial electron transport chain complex III, to reduce the production of ROS (22), and found that the AMPH-exaggerated caspase-3 activation and DNA damage during hypoxia were both abrogated (Figure $7 \mathrm{~F}$ ).

Taken together, our findings suggest a model whereby AMPH subverts the protective effect of HIF $1 \alpha$ in PAECs by PP-mediated lowering of p-Akt resulting in stabilization of SIRT1, an NAD Nependent $^{+}$ deacetylase. As a consequence, SIRT1 deacetylates and degrades HIF1 $\alpha$, causing an increase in oxidative phosphorylation and preventing the HIF $1 \alpha$-mediated COX4 isoform switch. As a result there is impaired mitochondrial electron transport resulting in excess mitochondrial ROS production. This induces the opening of MPTP and leakage of cytochrome $c$, fueling caspase-3 activation and DNA damage via caspaseactivated DNases (CADs), as described previously (38) (schema in Figure 8).

Pulmonary arteries of mice exposed to hypoxia and METH exhibit DNA damage. We next determined whether AMPH-exaggerated DNA damage during hypoxia would adversely affect vascular remodeling and worsen experimental pulmonary hypertension in a murine model. C57/BL6 mice were injected intraperitoneally with vehicle (saline) or METH (10 mg/kg) twice per day for 3 days, and then exposed to hypoxia $(10 \%$ $\mathrm{O}_{2}$ ) for 4 days. This regimen was repeated for 4 weeks. METH was used instead of AMPH because both showed similar results in cell culture studies described above and pharmaceutical grade was available for METH, but not AMPH. Compared with vehicle-injected mice, METH significantly increased heart rate and reduced pulmonary artery acceleration time (PAAT), consistent with worsening pulmonary hypertension (Supplemental Figure 4). Although there was not a significant increase in right ventricular systolic pressure (RVSP) or in right ventricle hypertrophy (RVH) with METH (Supplemental Figure 4), pulmonary vascular remodeling was more severe in the METH-treated mice, as judged by an increase in muscularized distal precapillary alveolar duct and wall arteries evident by $\alpha$-smooth muscle actin ( $\alpha$-SMA) staining (Figure 9A). Moreover, $\gamma \mathrm{H} 2 \mathrm{AX}$ foci were more prominent in the pulmonary arteries of METH-treated 
A
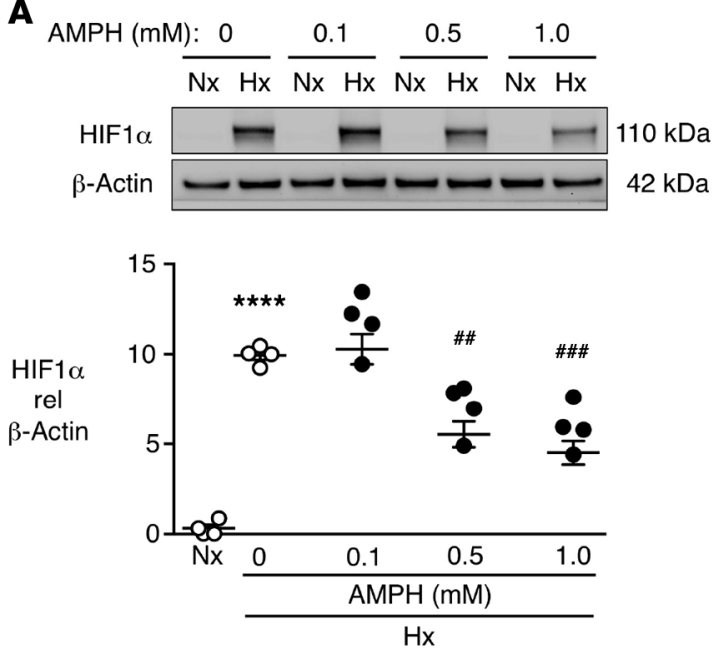

C

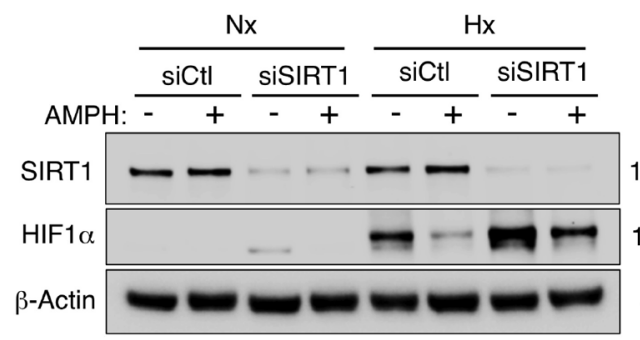

$120 \mathrm{kDa}$

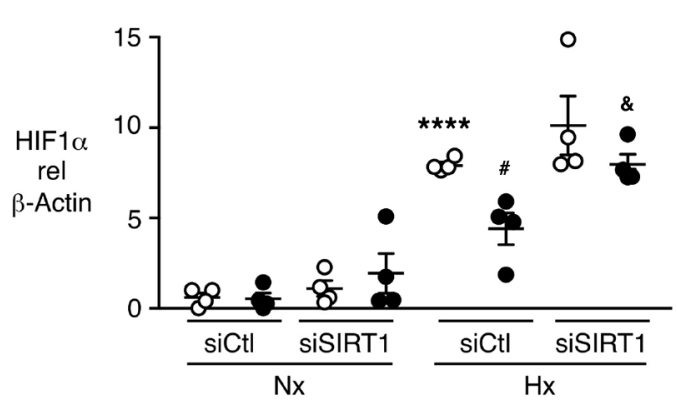

E

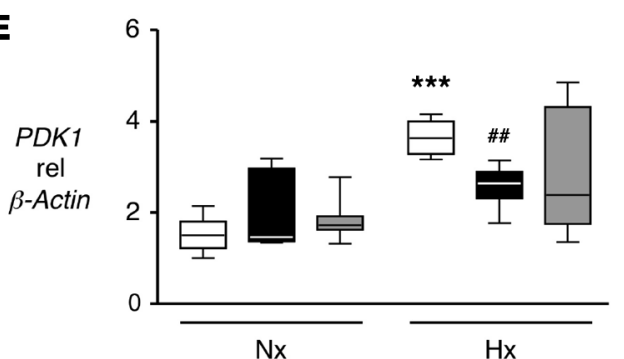

$\mathbf{F}$

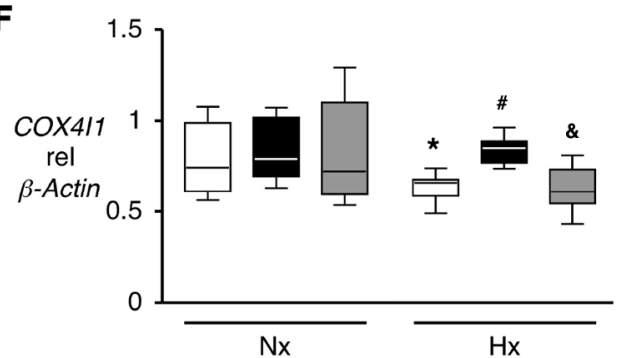

$110 \mathrm{kDa}$

$42 \mathrm{kDa}$

B

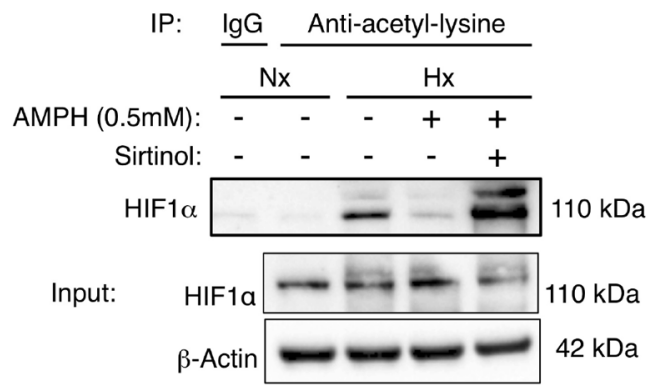

D

$\square$ Vehicle

口 AMPH (0.5mM)

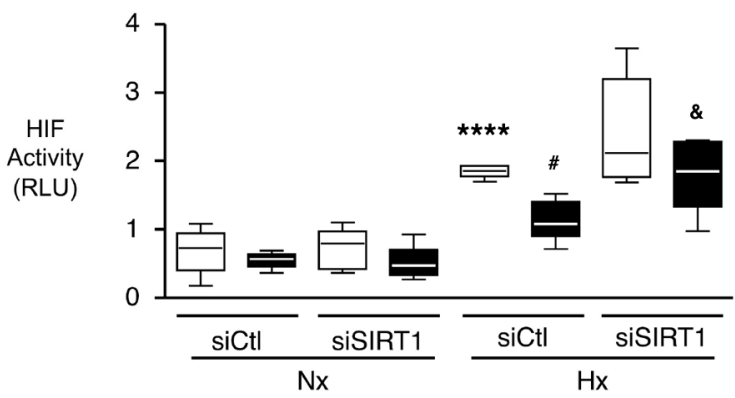

G

O Vehicle
AMPH $(0.5 \mathrm{mM})$

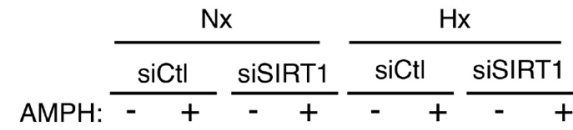

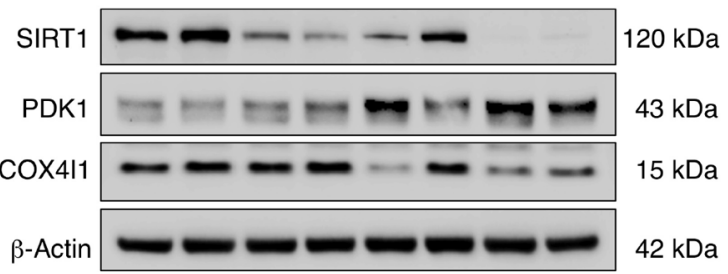

$\square$ Vehicle

AMPH $(0.5 \mathrm{mM})$

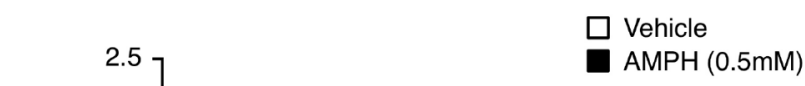

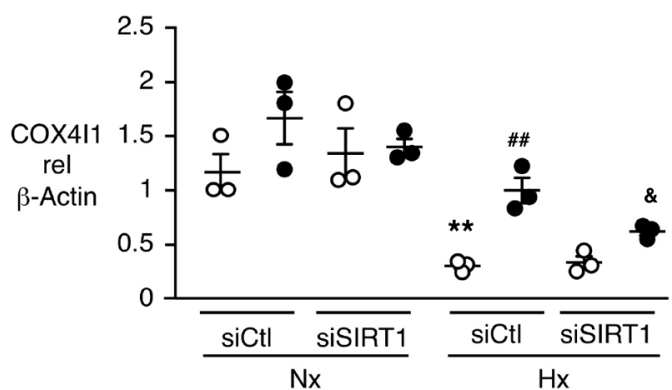

Figure 6. Stabilization of SIRT1 by amphetamine deacetylates and destabilizes HIF1 $\alpha$, impairing its transcriptional activity. (A) Pulmonary artery endothelial cells (PAECs) were treated daily with vehicle (Veh) or the indicated dose of amphetamine (AMPH) for 3 days and then continued with vehicle or AMPH under normoxia (Nx) or hypoxia ( $\mathrm{Hx}$ ) for 48 hours. Cell lysates were immunoblotted for HIF1 $\alpha$ and $\beta$-actin (for normalization). (B) PAECs were 
treated daily with $0.5 \mathrm{mM} \mathrm{AMPH}$ or AMPH with sirtinol $(10 \mu \mathrm{M})$ for 3 days, and then cultured with the same stimuli under Nx or Hx for 24 hours. Cell lysates were immunoprecipitated with anti-acetyl-Iysine antibody or IgC as negative control. Immunoprecipitates were probed for HIF1 $\alpha$ to evaluate the levels of acetylated HIF1 $\alpha$. Whole-cell lysates were immunoblotted to determine the levels of total HIF1 $\alpha$ and $\beta$-actin (loading control). A representative experiment is shown; 3 independent experiments were conducted with similar results. (C) PAECs were transfected with control or SIRT1 siRNAs, treated as in A and analyzed for HIF1 $\alpha$ levels. (D) Cignal HIF firefly luciferase reporter and Renilla luciferase plasmids were cotransfected with control or SIRT1 siRNAs into PAECs. Twenty-four hours after transfection, cells were treated with $0.5 \mathrm{mM}$ AMPH as in A and assessed for HIF activity, shown as the ratio of Firefly- to Renilla-luciferase-catalyzed light emission. (E and F) PAECs were treated as in B but with only 8 hours of Nx/Hx; RNA was extracted, and PDK1 and COX4I1 mRNA evaluated by qRT-PCR. (G) PAECs transfected and treated as in $\mathbf{C}$ were analyzed for PDK1 and COX4I1 protein levels. In $\mathbf{C}$ and $\mathbf{G}$, $\beta$-actin was probed as a loading control, and SIRT1 to verify SIRT1 knockdown efficiency. (A, C, and G) Dot plots represent mean \pm SEM, $n=3-4$. (D, E, and F) Box-and-whisker plots represent values within the interquartile range (boxes) and the minimum to maximum (whiskers). The line within the box shows the median. $n=3$ independent experiments with 2 to 3 replicates per experiment. ${ }^{*} P<0.05$, ${ }^{* *} P<0.005,{ }^{* * *} P<0.0005$, ${ }^{* * * *} P<0.0001 \mathrm{vs}$. Nx+Veh or siCtl+Nx+Veh; ${ }^{\#} P<0.05,{ }^{\#} P<0.005$, \#\#\# $P<0.0005$ vs. Hx+Veh or siCtl+Hx+Veh; ${ }^{P} P 0.05$ vs. Hx+AMPH or siCtl+Hx+AMPH; by 2 -way ANOVA, Bonferroni's post-test.

mice when compared with vehicle-treated mice under hypoxia (Figure 9B). Lung homogenates of METHtreated mice also showed increased $\alpha$-SMA (Figure 9C), and, consistent with the studies in PAECs, we documented reduced p-Akt, HIF1 $\alpha$, and PDK1, and increased SIRT1, COX4I1, and cleaved caspase-3 (Figure 9C), although we do not know whether these changes are localized selectively to the endothelial cells. It would be interesting to determine whether blocking these changes will reverse the increased muscularization seen with METH that we have related to the heightened DNA damage.

\section{Discussion}

This study uncovers a mechanism by which AMPH subverts a normal adaptive response to an environmental stress, and in so doing, impairs cellular function by inducing caspase-3 activation and DNA damage. Recent studies have shown that CAD can trigger DNA strand breaks without causing apoptosis $(38,39)$. This indicates that the cells can survive and accumulate damaged DNA as seen in Figure 1, and this might facilitate the mesenchymal transformation of PAECs previously described as a feature of PAH (40). Transformation of PAECs could impair their ability to repress smooth muscle cell (SMC) proliferation, accounting for the more severe muscularization in the METH-treated hypoxic murine model, and the pathology observed in clinical PAH. We chose to investigate the interaction of AMPH with hypoxia since hypoxia is a common cellular stress that occurs with respiratory disease, during sleep apnea, or even travel to high altitude. The AMPH-mediated amplification of DNA damage also would be expected with a variety of genotoxic agents, as we show with Dox.

Increased caspase-3 activation by AMPH under serum withdrawal or hypoxia is dependent on a severe reduction of Akt activity. Inhibition of p-Akt by AMPH was previously attributed to formation of a complex of Akt, PP2A, and $\beta$-arrestin2 (26). Although we found increased interaction of Akt and PP2A under hypoxia when treated with AMPH (data not shown), suppressing $\beta$-arrestin 2 or its family member $\beta$-arrestin 1 by siRNA did not reverse p-Akt inhibition (Supplemental Figure 5 ). This suggests that in our experimental system, AMPH may activate PP2A directly, without engaging the $\beta$-arrestin platform. Interestingly, recent studies have implicated several psychotropic small compounds commonly used to treat mental health conditions as potential direct activators of $\mathrm{PP} 2 \mathrm{~A}(41,42)$.

During the course of these studies, we investigated two other transcription factors, Forkhead family member FOXO3a and p53, by which AMPH inhibition of p-Akt could lead to amplified capase-3 activation and DNA damage under hypoxia (43). Reduced phosphorylation of FOXO3a due to p-Akt inhibition could induce its nuclear translocation and transcription of proapoptotic members of the BCL2-family. While AMPH did repress FOXO3a phosphorylation under both normoxia and hypoxia, this was not rescued by myr-Akt (Supplemental Figure 6), making its involvement in AMPH-exaggerated caspase-3 and DNA damage unlikely. p-Akt also phosphorylates murine double minute (Mdm2), increasing p53 ubiquitination and degradation. We found elevated phosphorylation of $\mathrm{p} 53$ by AMPH under hypoxia that was reversed by myr-Akt (Supplemental Figure 6). However, suppressing p53 by siRNA enhanced caspase-3 cleavage and DNA damage (data not shown), a response consistent with other studies by our group indicating that $\mathrm{p} 53$ is a survival factor in PAECs (22).

To associate AMPH-mediated p-Akt inhibition with aberrant epigenetic changes that lead to the exaggeration of caspase- 3 activation and DNA damage, we screened for differential expression of HDACs. We reasoned that deacetylation of histones by AMPH and hypoxia might enhance DNA damage by preventing access of chromatin repair proteins. Moreover, HDACs have been implicated in the long-lasting changes in the brain's reward circuitry generated by abuse of addictive drugs, such as 
A

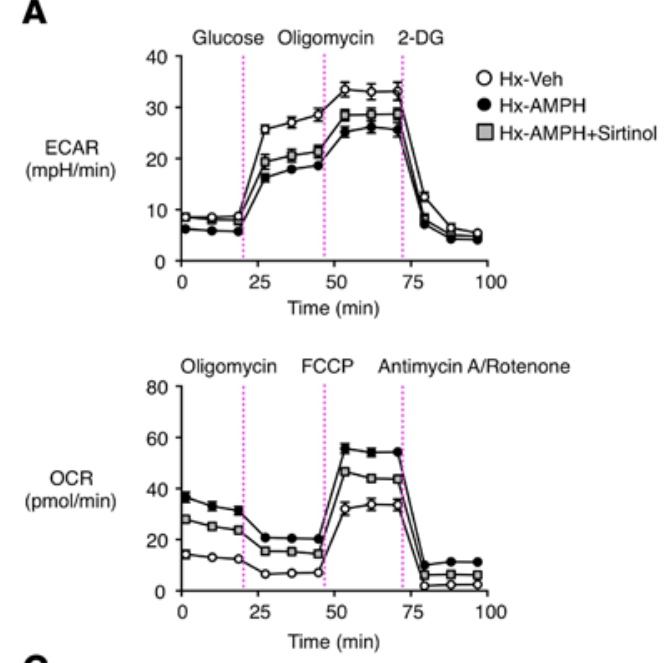

C
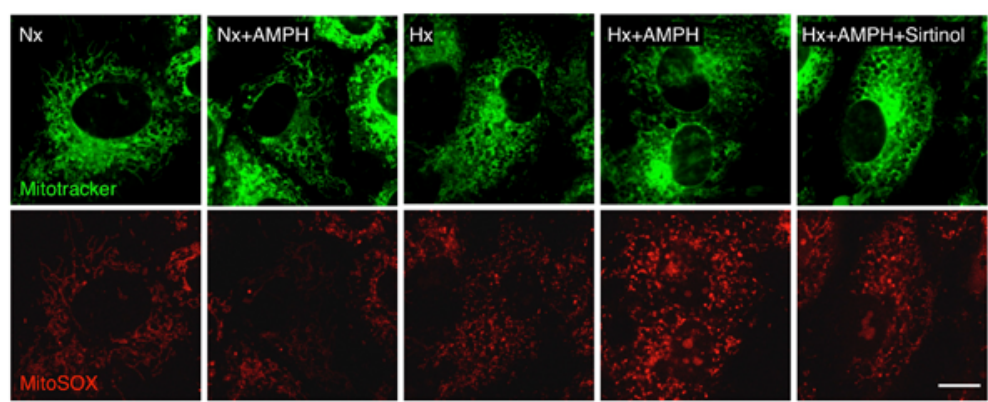

D
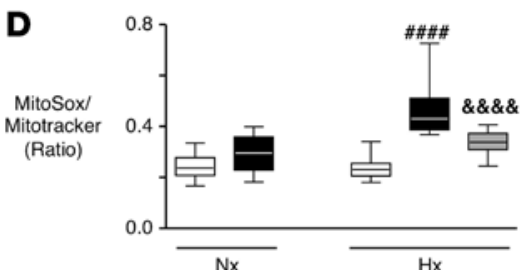

E

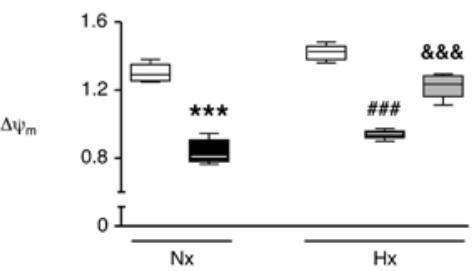

B
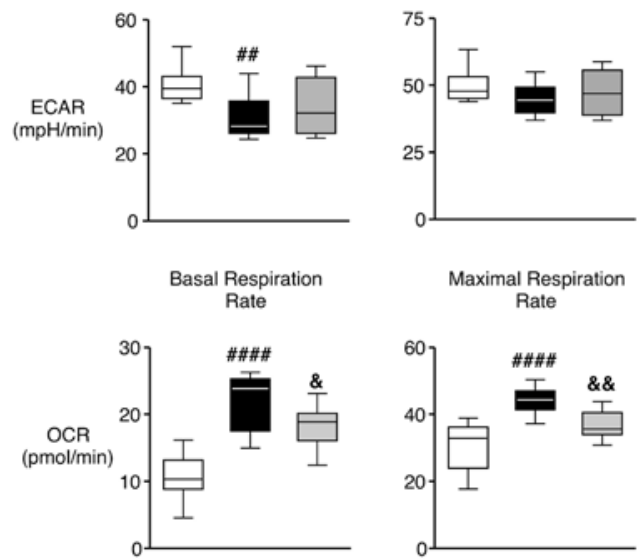

Maximal Respiration Rate

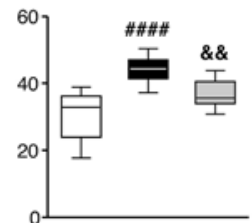

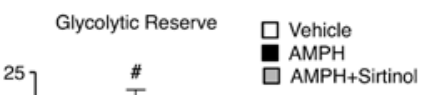

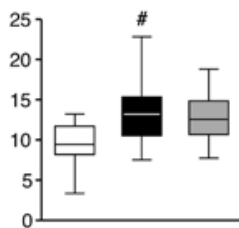

Spare Respiratory Capacity

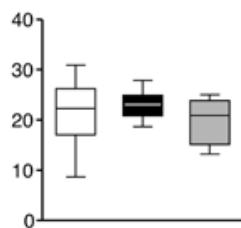

$\mathbf{F}$
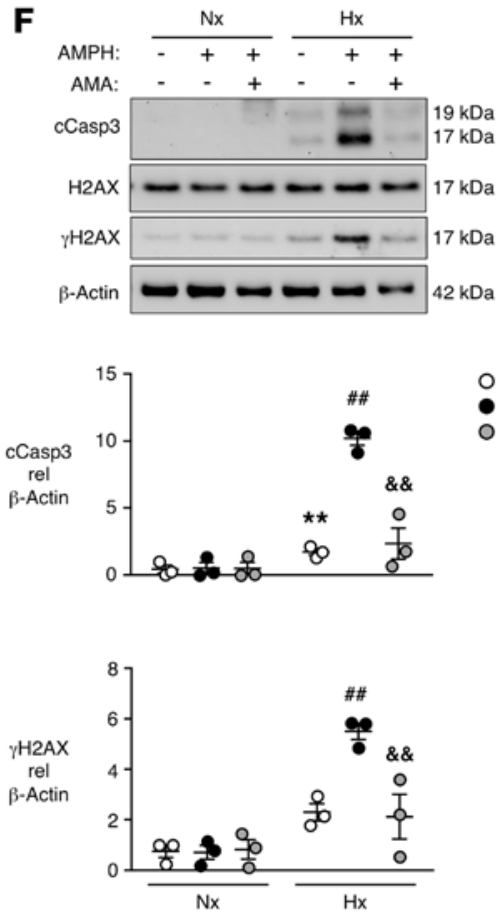

Figure 7. In PAECs under hypoxia, amphetamine impairs the transition from oxidative phosphorylation to glycolysis. (A) Pulmonary artery endothelial cells (PAECs) were treated with vehicle (Veh), $0.5 \mathrm{mM}$ amphetamine (AMPH), or AMPH with $10 \mu \mathrm{M}$ sirtinol, and placed in hypoxia (Hx) for 24 hours with the stimuli. Extracellular acidification rates (ECARs) and oxygen consumption rates (OCRs) were measured using kits (Seahorse Bioscience). (B) Glycolysis, glycolytic reserve, and glycolytic capacity as well as baseline respiration, maximal respiration, and spare respiratory capacity. (C) Representative live-cell images of PAECs treated as in A, loaded with $100 \mathrm{nM}$ MitoTracker Green FM to stain mitochondria, and $5 \mu \mathrm{M}$ MitoSOX Red to detect mitochondrial superoxide. Scale bar: $10 \mu \mathrm{m}$. (D) Fluorescence intensities of MitoTracker and MitoSOX were quantified using ImageJ, and ROS production was expressed as the ratio of MitoSOX to MitoTracker fluorescence. (E) PAECs were treated as in A. Mitochondrial membrane potential was determined using the JC-1 dye assay. (F) PAECs were treated with $0.5 \mathrm{mM} \mathrm{AMPH}$, or AMPH with $10 \mu \mathrm{M}$ of antimycin $\mathrm{A}$ (AMA), and then cultured with the stimuli under normoxia (Nx) or Hx for 48 hours. Cell lysates were immunoblotted for $\gamma \mathrm{H} 2 \mathrm{AX}$, cCasp3, $\beta$-actin, and H2AX (loading controls). (A and F) Line and dot plots represent mean \pm SEM, $n=3$ independent experiments. (B, D, and E) Box-and-whisker plots represent values within the interquartile range (boxes) and the minimum to maximum (whiskers). The line within the box shows the median. $n=3$ independent experiments with 3 to 4 replicates per experiment. ${ }^{* *} P<0.005,{ }^{* * *} P<0.0005$ vs. Nx+Veh; ${ }^{\#} P<0.05$, ${ }^{\# \#} P<0.005$, \#\#\# $P$

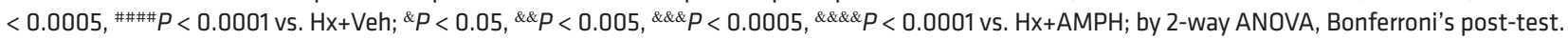

AMPH (METH) and cocaine (44). While several HDACs were upregulated by AMPH under hypoxia, we began by investigating the role of sirtuins, a protein family of metabolic sensors that translate changes in $\mathrm{NAD}^{+}$levels into adaptive responses under nutrient or energy stresses (45). Deacetylation by SIRT1 can lead to direct activation or inhibition of various transcriptional regulators and modify their 

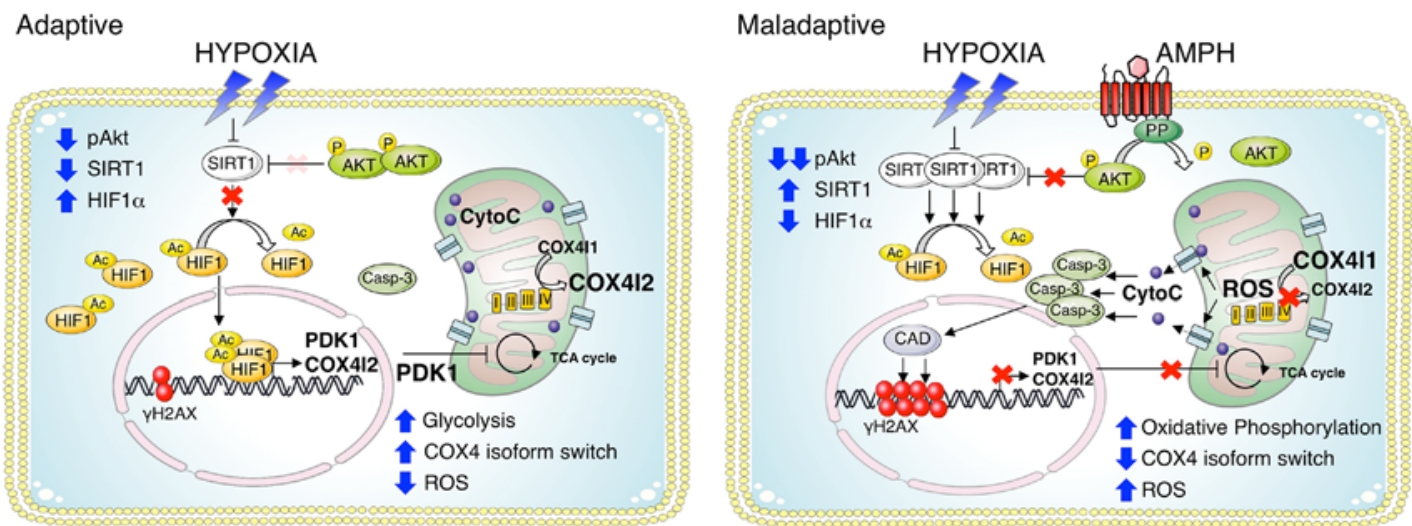

Figure 8. Proposed model. Under hypoxia, stabilization of HIF1 $\alpha$ is augmented by acetylation due to decreased sirtuin 1 (SIRT1) activity. HIF1 $\alpha$ activates pyruvate dehydrogenase kinase 1 (PDK1) expression and cytochrome $c$ oxidase 4 (COX4) isoform switch, thereby facilitating the transition from oxidative phosphorylation to glycolysis and optimizing electron transport during hypoxia. Elevation of SIRT1 by amphetamine (AMPH) under hypoxia, due to protein phosphatase (PP)mediated lowering of p-Akt, suppresses HIF1 $\alpha$-mediated PDK1 expression and the COX4 isoform switch, thereby increasing mitochondrial ROS production, cytochrome $c$ (CytoC) leakage, caspase-3 (Casp-3) activation, and ultimately DNA damage via caspase-activated DNases (CADs).

interaction profiles, depending on the cellular context. In addition to HIF1 $\alpha$, p53, and FOXO3a, SIRT1 is known to deacetylate PGC1 $\alpha$, all key regulators of metabolism, stress resistance, and apoptosis (45).

SIRT1-mediated deacetylation of HIF1 $\alpha$ activity led to increased oxidative metabolism, which under hypoxia resulted in accumulation of ROS and subsequent activation of caspase-3. Although SIRT1 is an enzyme that generally improves oxidative metabolism and mitochondrial function, heightened SIRT1 activity under certain conditions can be metabolically disadvantageous. In addition to the hypoxia context described in this report, constitutive activation of SIRT1 in the pancreas is detrimental for global glucose tolerance (46). SIRT1-deacetylated FOXO1 suppressed the expression of genes critical for pancreatic function and proper $\beta$ cell growth. Likewise, although SIRT1 protects against oxidative stress and ischemia/ reperfusion in cardiac muscle (47), enhanced SIRT1 activity together with peroxisome proliferator-activated receptor $\alpha(\mathrm{PPAR} \alpha)$ suppresses estrogen-related receptor target genes, thereby impairing energy production and contractility during pressure overload, and facilitating the progression of heart failure $(48,49)$.

In the context of healthy endothelial cells, we showed that induction of HIF $1 \alpha$ is adaptive and that the COX4 isoform switch prevents excess production of mitochondrial ROS, caspase activity, and DNA damage. This is not necessarily at odds with work showing that preventing HIF1 $\alpha$-mediated glycolysis by PDK1 inhibition with dichloroacetate has a beneficial effect in multiple experimental models including chronic hypoxia (50). Nor is it inconsistent with studies showing that HIF1 $\alpha$ heterozygous mice have attenuated chronic hypoxic pulmonary hypertension. We speculate that the marked destabilization of HIF1 $\alpha$ with METH during hypoxia not only prevents PDK1-mediated glycolysis but also the COX4 isoform switch, which is still operational with PDK inhibition and with HIF1 $\alpha$ heterozygosity (51). In the D\&T-PAH vs. control PAECs, there is similar caspase activation in response to hypoxia and AMPH, associated with lowering of p-Akt and HIF1 $\alpha$ and no evidence of a COX4 isoform switch. This suggests that AMPH causes the cumulative DNA damage in these cells, consistent with the comet assay in Figure 1. It would be interesting to assess whether in pulmonary artery SMCs or fibroblasts, in contrast with PAECs, AMPH heightens the proliferative response to hypoxia.

AMPH-induced DNA damage during hypoxia was recapitulated in the pulmonary vasculature of the mouse model we used. Although the METH- versus vehicle-treated hypoxic mice showed increased pulmonary arterial remodeling and reduced PAAT, the RVSP was not significantly higher in the AMPH- than in the vehicle-treated group. Similar results were observed previously in the rat, with worse pulmonary arterial remodeling in response to METH but little increase in pulmonary arterial pressure (52). It is possible that the acute effect of METH may mask hypoxia-induced vasoconstriction, or alternatively, that the DNA damage induced by METH was not sufficient to cause more severe disease manifest by an elevation in RVSP. Longer duration of AMPH exposure and more cumulative DNA damage may be necessary to 

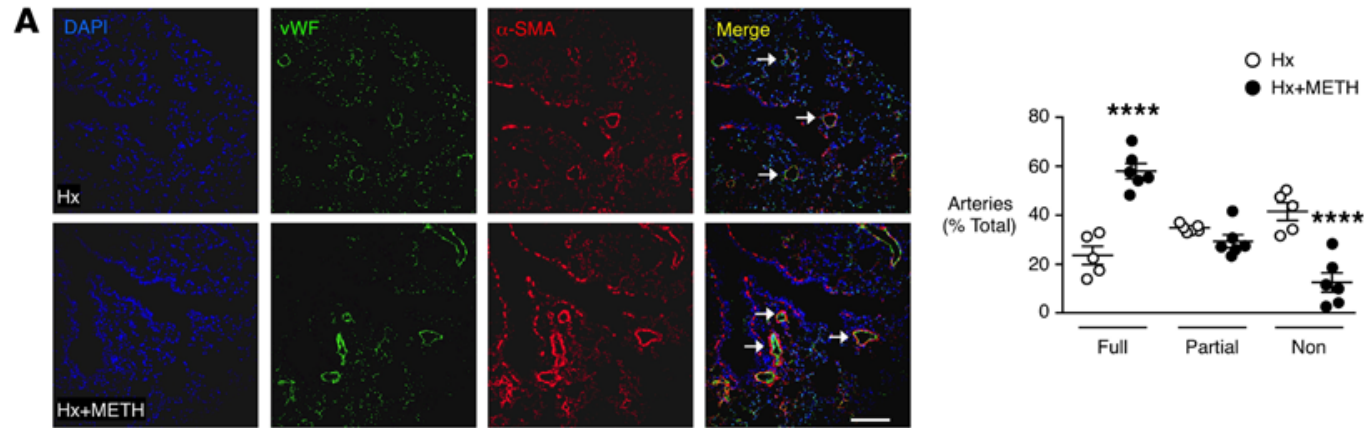

B

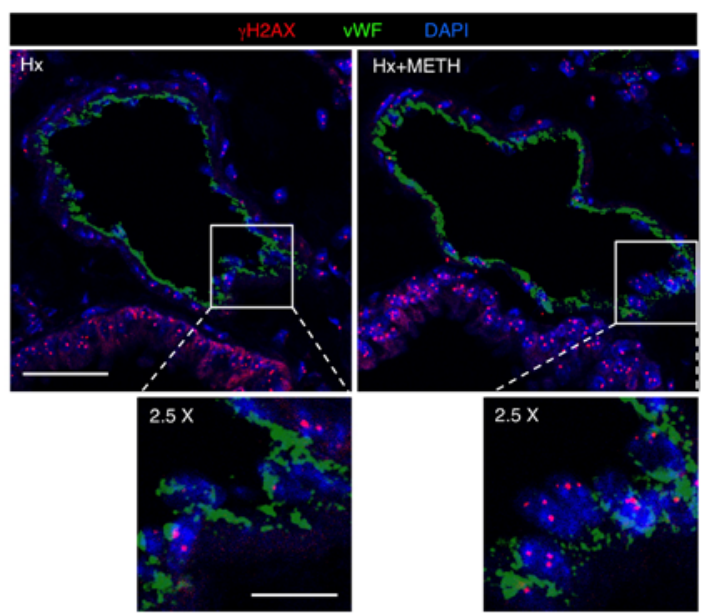

C

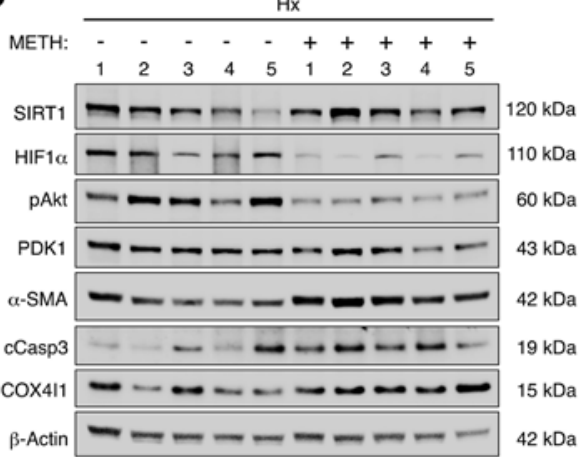

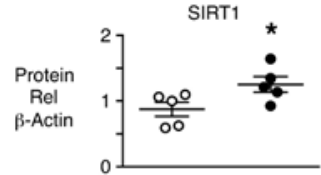
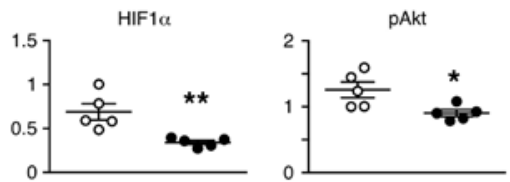

$\mathrm{OHx}$

- $\mathrm{Hx}+\mathrm{METH}$

PDK1
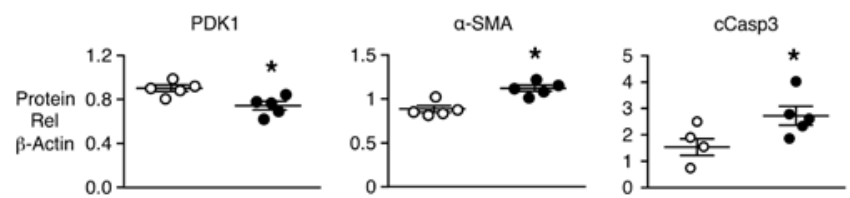

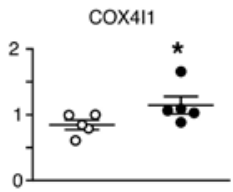

Figure 9. Methamphetamine and hypoxia increase $\gamma \mathrm{H} 2 \mathrm{AX}$ foci in mouse pulmonary arteries (PAs) and pulmonary vascular remodeling and impaired HIF1 $\alpha$ gene regulation. Mice were treated with $10 \mathrm{mg} / \mathrm{kg}$ methamphetamine (METH) twice daily for 3 days in room air, and then exposed to $10 \% \mathrm{O}_{2}$ hypoxia for 4 days, and the cycle repeated for a total of 4 weeks. (A and B) Mouse lung sections were immunostained for von Willebrand factor (vWF, green), DAPI (blue), and $\alpha$-smooth muscle actin ( $\alpha$-SMA) (A) or $\gamma \mathrm{H} 2 \mathrm{AX}$ (B) (red), to indicate the PA endothelial cell layer, nuclei, muscularization, and DNA damage foci, respectively. (A) Representative images of muscularized distal PAs (DPAs), indicated by arrows. Scale bar: $80 \mu \mathrm{m}$. Scatter plot on the right shows the percentage of fully, partially, and nonmuscularized DPAs, scored based on 4 to 5 confocal images taken for each mouse. (B) Representative images of PAs immunostained for $\gamma \mathrm{H} 2 \mathrm{AX}$. Scale bar: $20 \mu \mathrm{m}$. Insets show magnified areas of endothelial cells with $\gamma \mathrm{H} 2 \mathrm{AX}$ foci. Scale bar; $8 \mu \mathrm{m}$. Right, $\gamma \mathrm{H} 2 \mathrm{AX}$ foci were scored using Image), in 10 to 15 confocal images of PAs for each mouse. (C) Lung homogenates from the mice were immunoblotted for SIRT1, HIF1 $\alpha$, p-Akt, PDK1, $\alpha$-SMA, PDK1, cCasp3, COX4I1, and $\beta$-actin (loading control). Each lane represents lung lysate of one mouse. Dot plots in $\mathbf{A}-\mathbf{C}$ represent mean \pm SEM; $n=5$, vehicle-treated (Veh) group and $n=5-6$, METH group. ${ }^{*} P<0.05,{ }^{* *} P<0.005,{ }^{* * *} P<0.0001$ vs. vehicle by unpaired $t$ test.

induce more severe disease. Alternatively, factors additional to DNA damage may be required. These could include epigenetic modifications resulting from excess mitochondrial ROS production (53).

Modeling the PAH response to AMPH/METH is difficult in rodents despite the similarities that we showed in the molecular pathway. Because there are significant differences in METH elimination half-lives in the various mammalian species, it has been suggested that the drug should be given every 30 minutes to rodents to better represent the 3-hour intervals of self-administration that some human METH addicts use during binges (54). In addition, patients present with PAH only after many years of AMPH/METH abuse. 
Table 2. Characteristics of Donor Controls that were the source of PAECs used in the experiments in Figure 1

\begin{tabular}{lccccc}
\hline ID & PAEC Type & Age (yr) & Gender & Race/Ethnicity & Cause of death \\
Ctl-1 & LPAEC & 57 & F & White/Non-Hispanic & Acute Myocardial Infarction \\
Ctl-2 & LPAEC & 50 & F & Hispanic or Latino & Cerebrovascular/Stroke \\
CtI-3 & LPAEC & 33 & F & White/Non-Hispanic & Head trauma. Blunt injury. \\
CtI-4 & LPAEC & 49 & F & Black or African American/Non-Hispanic & Cerebrovascular/Stroke, intracranial hemorrhage \\
PAEC, pulmonary artery endothelial cell; LPAEC, large PAEC. &
\end{tabular}

It is interesting that even with a significant period of AMPH/METH abstinence prior to transplant, the DNA damage persisted in endothelial cells cultured from these lung explants. This may indicate that the DNA repair mechanism is also impaired.

As we have implicated mitochondrial dysfunction as the source of caspase activation and DNA damage, it would be interesting to investigate whether factors such as succinate dehydrogenase could be involved $(55,56)$ or whether agents such as metformin could reverse the ROS-mediated DNA damage, as we showed with antimycin (57).

Since not all users of anorexigens or AMPH develop PAH, researchers have speculated that a second environmental perturbation and/or a genetic predisposition is involved in promoting the adverse pulmonary vascular effects of these agents. Our study shows how AMPH can subvert the normal cellular response to an environmental stress (i.e. hypoxia), causing perturbation of mitochondrial function, heightened production of ROS, caspase-3 activation, and DNA damage. It is likely that PAH occurs with AMPH when these features are either cumulative over time or further aggravated by other genetic features that could predispose to slower metabolism and higher levels of AMPH (58), reduced function of DNA repair proteins such as TopBP1 (21), or vulnerability to apoptosis induced by loss of function of BMPR2 (22).

\section{Methods}

Extended experimental procedures are provided in the Supplemental Information.

Human PAECs. Primary human PAECs were commercially obtained from PromoCell GmbH. Additional primary PAECs used in the studies in Figure 1 were obtained from lungs removed at transplantation from PAH patients, and from unused donor lungs as controls, provided by the Pulmonary Hypertension Breakthrough Initiative (PHBI) network and Stanford (patient and donor characteristics in Tables 1 and 2). PAECs were grown in Endothelial Cell Medium (ScienCell) with growth supplements and used between passages 4 and 7. In the studies examining the AMPH-exaggerated DNA damage responses, human PAECs were refreshed daily with AMPH in full medium for 3 days and then exposed to genotoxic stress (Dox or normoxia/hypoxia) with the same stimuli for indicated times.

Mouse model. C57BL/6 mice (6-8 weeks old, male) were obtained from Charles River and housed in the Animal Facility at Stanford, with food and water ad libitum. METH (pharmaceutical grade methamphetamine hydrochloride, United States Pharmacopeial Convention) was dissolved in sterile saline and intraperitoneally administered $(10 \mathrm{mg} / \mathrm{kg})$ twice daily for 3 days, followed by 4 days in hypoxia $\left(10 \% \mathrm{O}_{2}\right)$. This regimen was repeated for a total of 4 weeks. Vehicle-treated animals were injected with an equal volume of sterile saline. RVSP, RVH, cardiac function and output, and PAAT were measured as described previously (59). For tissue studies, the heart and lungs were perfused with PBS, the left lung fixed, and sections embedded in paraffin for immunohistochemistry and morphometry. The right lung was snap-frozen in liquid $\mathrm{N}_{2}$ and kept at $-80^{\circ} \mathrm{C}$ for protein extraction. The number of mice per experiment is indicated in the figure legends.

Reagents and cellular assays. Commercial reagents and kits used for cellular assays are described in the Supplemental Information.

Comet assay. DNA breaks were monitored using the Comet Assay Reagent Kit for Single Cell Gel Electrophoresis Assay (Trevigen) according to the manufacturer's protocol. DNA was stained with SYBR-green (Invitrogen). The percentage of DNA in the comet tail was measured with ImageJ (NIH) in 100 to 150 cells for each sample.

HIF reporter assay. PAECs were transfected with HIF-responsive luciferase constructs that encode the firefly luciferase reporter gene under the control of the HIF response elements (Cignal HIF Pathway 
Reporter Assay Kit, Qiagen). A Renilla construct encoding the Renilla luciferase reporter gene under the control of a CMV promoter was cotransfected as an internal control to normalize transfection efficiency and monitor cell viability.

Caspase assay. PAECs treated with indicated reagents for 24 hours in serum-free medium were incubated for 1 hour in caspase-3/7 luciferase reagent mix, and total luminescence was measured in a plate reader.

Mitochondrial metabolism. Baseline mitochondrial function and mitochondrial stress response were evaluated by OCR using the Cell Mito Stress Kit with an XF24 extracellular flux analyzer (Seahorse Bioscience). Glycolytic capacity was measured using the XF Glycolysis Stress Test Kit (Seahorse Bioscience) on the same device.

Mitochondrial membrane potential assay. The mitochondrial membrane potential was determined using the JC-1 dye (5',6,6'-tetrachloro-1,1',3,3'-tetraethylbenzimidazolylcarbocyanine iodide) assay (Immunochemistry Technologies).

Mitochondrial ROS assay. PAECs seeded onto live-cell imaging glass-bottom dishes were loaded with 100 nM MitoTracker Green FM (Molecular Probes) to stain mitochondria and $5 \mu \mathrm{M}$ MitoSOX Red (Molecular Probes) to detect mitochondrial superoxide. ROS production was normalized to cell number and mitochondrial content, and calculated as the ratio of MitoSOX to MitoTracker fluorescence.

siRNA transfection. siRNA specific for human SIRT1 or siControl was transfected into PAECs by nucleofection. Knockdown efficiency was determined by immunoblotting and qRT-PCR.

Immunofluorescence. Sections from formaldehyde-fixed, paraffin-embedded mouse lungs were deparaffinized and rehydrated. Epitope retrieval was performed, and sections were then incubated with the primary antibodies listed in the Supplemental Information against $\alpha$-SMA, $\gamma \mathrm{H} 2 \mathrm{AX}$, and von Willebrand factor (vWF).

Western immunoblotting. Total proteins were extracted from cells or tissues with SDS or CHAPS lysis buffer, respectively, and analyzed as previously described (40). $\beta$-Actin or GAPDH was used for normalization/ loading control, as indicated in the legends. Antibodies used are indicated in the Supplemental Information.

qRT-PCR. Total RNA was extracted and purified from cells (Zymo Research). The quantity and quality of RNA were determined by a spectrophotometer, and then RNA was reverse transcribed to cDNA (60). The primers are listed in the Supplemental Information.

Statistics. All data are expressed as arithmetical mean \pm SEM. Statistical significance was determined by 1-way or 2-way ANOVA followed by Bonferroni post-test. Two-tailed unpaired $t$-test analysis was used for comparison of 2 groups. A $P$ value less than 0.05 was considered significant. Significance is shown only for relevant comparisons. The number of experiments, animals per group, and the statistical test used are indicated in the figure legends.

Study approval. The Animal Care Committee of Stanford University approved all protocols, in keeping with the regulations of the American Physiological Society. Procurement of the tissues from human subjects was approved by the Administrative Panel on Human Subjects in Medical Research at Stanford University (IRB number 350, Panel 6). Written informed consent was received from participants prior to inclusion in the study.

\section{Author contributions}

PIC designed and executed $90 \%$ of the experiments, analyzed the data, and wrote the manuscript. AC assisted with the development of the mouse model. KM and LW performed the hemodynamic and echocardiographic measurements, and NFT helped with monitoring the animals and morphometric assessments. JKH assisted with the mitochondrial function studies. CGL assisted with the comet assays. NMS and AI helped with DNA damage foci analysis in the mouse lung. DL helped with cell culture. MY assisted with the qRT-PCR analysis. MR was responsible for overall study design, oversight of experiments, data acquisition and analysis, and manuscript preparation.

\section{Acknowledgments}

We greatly appreciate the editorial assistance of Michal Bental Roof and the administrative help of Michelle Fox. PAECs from PAH and control patients were provided by the PHBI. Funding for the PHBI is provided under an NIH National Heart, Lung, and Blood Institute (NHLBI) grant R24 HL123767, and by the Cardiovascular Medical Research and Education Fund (CMREF). The hypoxia workstation was provided by Amato Giaccia (Stanford University). Studies were supported by NIH-NHLBI grants R01 HL087118 and R01 HL074186 and the Dunlevie Chair in Pediatric Cardiology (to M.R.). 
Address correspondence to: Marlene Rabinovitch, CCSR-1215A, 269 Campus Drive, Stanford, California 94305, USA. Phone: 650.723.6928; E-mail: marlener@stanford.edu.

JKH's current address is: Department of Medicine, University Medical Center Hamburg-Eppendorf, Hamburg, Germany.

NMS's current address is: Department of Pediatrics, University of California San Diego, San Diego, California, USA.

1. Cadet JL, Krasnova IN, Jayanthi S, Lyles J. Neurotoxicity of substituted amphetamines: molecular and cellular mechanisms. Neurotox Res. 2007;11(3-4):183-202.

2. Ramirez SH, et al. Methamphetamine disrupts blood-brain barrier function by induction of oxidative stress in brain endothelial cells. J Cereb Blood Flow Metab. 2009;29(12):1933-1945.

3. Yamamoto BK, Raudensky J. The role of oxidative stress, metabolic compromise, and inflammation in neuronal injury produced by amphetamine-related drugs of abuse. J Neuroimmune Pharmacol. 2008;3(4):203-217.

4. Andreazza AC, et al. Effects of mood stabilizers on DNA damage in an animal model of mania. J Psychiatry Neurosci. 2008;33(6):516-524

5. Imam SZ, Ali SF. Selenium, an antioxidant, attenuates methamphetamine-induced dopaminergic toxicity and peroxynitrite generation. Brain Res. 2000;855(1):186-191.

6. Hirata H, Ladenheim B, Carlson E, Epstein C, Cadet JL. Autoradiographic evidence for methamphetamine-induced striatal dopaminergic loss in mouse brain: attenuation in CuZn-superoxide dismutase transgenic mice. Brain Res. 1996;714(1-2):95-103.

7. Chin KM, Channick RN, Rubin LJ. Is methamphetamine use associated with idiopathic pulmonary arterial hypertension? Chest. 2006;130(6):1657-1663.

8. Schaiberger PH, Kennedy TC, Miller FC, Gal J, Petty TL. Pulmonary hypertension associated with long-term inhalation of "crank" methamphetamine. Chest. 1993;104(2):614-616.

9. Rothman RB, Ayestas MA, Dersch CM, Baumann MH. Aminorex, fenfluramine, and chlorphentermine are serotonin transporter substrates. Implications for primary pulmonary hypertension. Circulation. 1999;100(8):869-875.

10. Aldred MA, et al. Somatic chromosome abnormalities in the lungs of patients with pulmonary arterial hypertension. Am J Respir Crit Care Med. 2010;182(9):1153-1160.

11. Yeager ME, Halley GR, Golpon HA, Voelkel NF, Tuder RM. Microsatellite instability of endothelial cell growth and apoptosis genes within plexiform lesions in primary pulmonary hypertension. Circ Res. 2001;88(1):E2-E11.

12. Rabinovitch M. Molecular pathogenesis of pulmonary arterial hypertension. J Clin Invest. 2012;122(12):4306-4313.

13. Rothman RB, Baumann MH. Methamphetamine and idiopathic pulmonary arterial hypertension: role of the serotonin transporter. Chest. 2007;132(4):1412-1413.

14. de Jesus Perez V, Kudelko K, Snook S, Zamanian RT. Drugs and toxins-associated pulmonary arterial hypertension: lessons learned and challenges ahead. Int J Clin Pract Suppl. 2011(169):8-10.

15. Stenmark KR, Fagan KA, Frid MG. Hypoxia-induced pulmonary vascular remodeling: cellular and molecular mechanisms. Circ Res. 2006;99(7):675-691.

16. Long L, et al. Serotonin increases susceptibility to pulmonary hypertension in BMPR2-deficient mice. Circ Res. 2006;98(6):818-827. 17. Bristow RG, Hill RP. Hypoxia and metabolism. Hypoxia, DNA repair and genetic instability. Nat Rev Cancer. 2008;8(3):180-192.

18. Federici C, et al. Increased mutagen sensitivity and DNA damage in pulmonary arterial hypertension. Am J Respir Crit Care Med. 2015;192(2):219-228.

19. Meloche J, et al. Role for DNA damage signaling in pulmonary arterial hypertension. Circulation. 2014;129(7):786-797.

20. Li M, et al. Loss of bone morphogenetic protein receptor 2 is associated with abnormal DNA repair in pulmonary arterial hypertension. Am J Respir Cell Mol Biol. 2014;50(6):1118-1128.

21. de Jesus Perez VA, et al. Whole-exome sequencing reveals TopBP1 as a novel gene in idiopathic pulmonary arterial hypertension. Am J Respir Crit Care Med. 2014;189(10):1260-1272.

22. Diebold I, et al. BMPR2 preserves mitochondrial function and DNA during reoxygenation to promote endothelial cell survival and reverse pulmonary hypertension. Cell Metab. 2015;21(4):596-608.

23. Ross WE. DNA topoisomerases as targets for cancer therapy. Biochem Pharmacol. 1985;34(24):4191-4195.

24. Tallóczy Z, et al. Methamphetamine inhibits antigen processing, presentation, and phagocytosis. PLoS Pathog. 2008;4(2):e28

25. Volkow ND, et al. Distribution and pharmacokinetics of methamphetamine in the human body: clinical implications. PLoS One. 2010;5(12):e15269.

26. Beaulieu JM, Sotnikova TD, Marion S, Lefkowitz RJ, Gainetdinov RR, Caron MG. An Akt/beta-arrestin 2/PP2A signaling complex mediates dopaminergic neurotransmission and behavior. Cell. 2005;122(2):261-273.

27. Dinant C, Houtsmuller AB, Vermeulen W. Chromatin structure and DNA damage repair. Epigenetics Chromatin. 2008;1(1):9.

28. Perez-Perri JI, Acevedo JM, Wappner P. Epigenetics: new questions on the response to hypoxia. Int J Mol Sci. 2011;12(7):4705-4721.

29. Han L, Zhao G, Wang H, Tong T, Chen J. Calorie restriction upregulated sirtuin 1 by attenuating its ubiquitin degradation in cancer cells. Clin Exp Pharmacol Physiol. 2014;41(3):165-168

30. Gao Z, Zhang J, Kheterpal I, Kennedy N, Davis RJ, Ye J. Sirtuin 1 (SIRT1) protein degradation in response to persistent c-Jun N-terminal kinase 1 (JNK1) activation contributes to hepatic steatosis in obesity. J Biol Chem. 2011;286(25):22227-22234.

31. Abdelmohsen K, et al. Phosphorylation of HuR by Chk2 regulates SIRT1 expression. Mol Cell. 2007;25(4):543-557.

32. Sengupta S, Jang BC, Wu MT, Paik JH, Furneaux H, Hla T. The RNA-binding protein HuR regulates the expression of cyclooxygenase-2. J Biol Chem. 2003;278(27):25227-25233. 
33. Wu T, Shi JX, Geng S, Zhou W, Shi Y, Su X. The MK2/HuR signaling pathway regulates TNF- $\alpha$-induced ICAM-1 expression by promoting the stabilization of ICAM-1 mRNA. BMC Pulm Med. 2016;16(1):84

34. Nakagawa T, Guarente L. Sirtuins at a glance. J Cell Sci. 2011;124(Pt 6):833-838.

35. Lim JH, Lee YM, Chun YS, Chen J, Kim JE, Park JW. Sirtuin 1 modulates cellular responses to hypoxia by deacetylating hypoxia-inducible factor 1alpha. Mol Cell. 2010;38(6):864-878.

36. Semenza GL. Hypoxia-inducible factors in physiology and medicine. Cell. 2012;148(3):399-408.

37. Fukuda R, Zhang H, Kim JW, Shimoda L, Dang CV, Semenza GL. HIF-1 regulates cytochrome oxidase subunits to optimize efficiency of respiration in hypoxic cells. Cell. 2007;129(1):111-122.

38. Larsen BD, Rampalli S, Burns LE, Brunette S, Dilworth FJ, Megeney LA. Caspase 3/caspase-activated DNase promote cell differentiation by inducing DNA strand breaks. Proc Natl Acad Sci USA. 2010;107(9):4230-4235.

39. Orth JD, Loewer A, Lahav G, Mitchison TJ. Prolonged mitotic arrest triggers partial activation of apoptosis, resulting in DNA damage and p53 induction. Mol Biol Cell. 2012;23(4):567-576.

40. Hopper RK, et al. In pulmonary arterial hypertension, reduced BMPR2 promotes endothelial-to-mesenchymal transition via HMGA1 and its target Slug. Circulation. 2016;133(18):1783-1794.

41. Sangodkar J, Farrington CC, McClinch K, Galsky MD, Kastrinsky DB, Narla G. All roads lead to PP2A: exploiting the therapeutic potential of this phosphatase. FEBS J. 2016;283(6):1004-1024.

42. McClinch K, et al. Abstract C132: Therapeutic reactivation of PP2A for prostate cancer treatment. Molecular Cancer Therapeutics. 2015;14(12):Supplement 2.

43. Chong ZZ, Li F, Maiese K. Activating Akt and the brain's resources to drive cellular survival and prevent inflammatory injury. Histol Histopathol. 2005;20(1):299-315.

44. Renthal W, et al. Genome-wide analysis of chromatin regulation by cocaine reveals a role for sirtuins. Neuron. 2009;62(3):335-348.

45. Houtkooper RH, Pirinen E, Auwerx J. Sirtuins as regulators of metabolism and healthspan. Nat Rev Mol Cell Biol. 2012;13(4):225-238

46. Bai P, et al. PARP-2 regulates SIRT1 expression and whole-body energy expenditure. Cell Metab. 2011;13(4):450-460.

47. Hsu CP, et al. Silent information regulator 1 protects the heart from ischemia/reperfusion. Circulation. 2010;122(21):2170-2182

48. Kawashima T, et al. Constitutive SIRT1 overexpression impairs mitochondria and reduces cardiac function in mice. J Mol Cell Cardiol. 2011;51(6):1026-1036.

49. Oka S, et al. PPAR $\alpha$-Sirt1 complex mediates cardiac hypertrophy and failure through suppression of the ERR transcriptional pathway. Cell Metab. 2011;14(5):598-611.

50. Michelakis ED, et al. Dichloroacetate, a metabolic modulator, prevents and reverses chronic hypoxic pulmonary hypertension in rats: role of increased expression and activity of voltage-gated potassium channels. Circulation. 2002;105(2):244-250.

51. Yu AY, et al. Impaired physiological responses to chronic hypoxia in mice partially deficient for hypoxia-inducible factor 1alpha J Clin Invest. 1999;103(5):691-696

52. Wang Y, et al. Involvement of serotonin mechanism in methamphetamine-induced chronic pulmonary toxicity in rats. Hum Exp Toxicol. 2013;32(7):736-746.

53. Ziech D, Franco R, Pappa A, Panayiotidis MI. Reactive oxygen species (ROS)--induced genetic and epigenetic alterations in human carcinogenesis. Mutat Res. 2011;711(1-2):167-173.

54. Cadet JL, Jayanthi S, Deng X. Speed kills: cellular and molecular bases of methamphetamine-induced nerve terminal degeneration and neuronal apoptosis. FASEB J. 2003;17(13):1775-1788.

55. Mills E, O’Neill LA. Succinate: a metabolic signal in inflammation. Trends Cell Biol. 2014;24(5):313-320

56. Mills EL, et al. Succinate dehydrogenase supports metabolic repurposing of mitochondria to drive inflammatory macrophages. Cell. 2016;167(2):457-470.e13.

57. Algire C, et al. Metformin reduces endogenous reactive oxygen species and associated DNA damage. Cancer Prev Res (Phila). 2012;5(4):536-543.

58. de Jesus Perez VA, Orcholski M, Artyom K, Yuan K, Zamanian RT. B20 HOOKED ON PH'OMICS. Paper presented at: American Thoracic Society Conference; May 2016; Washington, DC.

59. Sawada H, et al. Reduced BMPR2 expression induces GM-CSF translation and macrophage recruitment in humans and mice to exacerbate pulmonary hypertension. J Exp Med. 2014;211(2):263-280.

60. Rhodes CJ, et al. RNA sequencing analysis detection of a novel pathway of endothelial dysfunction in pulmonary arterial hypertension. Am J Respir Crit Care Med. 2015;192(3):356-366. 